\title{
Rotation sets and Morse decompositions in twist maps
}

\author{
PHILIP L. BOYLAND \\ Department of Mathematics, Maharishi International University, \\ Fairfield, IA 52556, USA
}

\begin{abstract}
Positive tilt maps of the annulus are studied, and a correspondence is developed between the rotation set of the map and certain of its Morse decompositions. The main tool used is a characterization of fixed point free lifts of positive tilt maps. As an application, some alternative hypotheses under which the conclusions of the Aubry-Mather theorem hold are given, and it is also shown that the rotation band of a chain transitive set is always in the rotation set of the map.
\end{abstract}

\section{Introduction}

A fundamental idea in Conley's work is the decomposition of a dynamical system into its chain recurrent and gradient-like parts. The study of the system is thereby split into two pieces based on the asymptotic behaviour of orbits. In maps of the annulus, one aspect of this asymptotic behaviour is measured using the rotation number (or, more generally, the rotation set) of an orbit. It is natural to ask about the relationship of these two notions. For example, what does the set of rotation numbers of a map tell one about its dynamics or, more specifically, about its chain recurrent/gradient-like decomposition? A related question is: given a number in the rotation set of a map, is there always a nice recurrent set (for example, a minimal set) with that rotation number? Looking at things from the other direction, what type of information about the rotation set of a map can one derive from dynamical hypothesis on the map, for example, from the existence of certain types of invariant sets?

If the map of the annulus happens to be an area-preserving monotone twist map (and thus all chain recurrent), the Aubry-Mather theorem provides very clear answers to certain of these questions. In this case the set of rotation numbers is precisely that expected (i.e. all the numbers between the rotation numbers of the map restricted to the boundary circles), and for each number in this set the map has a well understood minimal set with that rotation number. If, on the other hand, the map is only assumed to be a homeomorphism, Franks [19] provides an answer to certain of these questions, but the general situation is still unclear. Franks shows that for each rational in the rotation set the map has a periodic orbit with that rotation number (this result was also obtained by Handel [22]). In addition, the existence of certain chain transitive sets and certain types of orbits implies the existence of a collection of periodic orbits whose rotation numbers are dense in an interval. 
In this paper we focus on a case that lies between these two extremes, closer to the former but using techniques closer to those of the latter. We study positive tilt maps of the annulus (a generalization of monotone twist studied in [21], [24] and [29]), but without any assumption of preserving area. In this case the set of rotation numbers of the map $f$, denoted $\rho(f)$, is closed and there is a nice correspondence between $\rho(f)$ and Conley's decomposition. Very roughly, the connected components of $\rho(f)$ correspond to the chain recurrent part and the open intervals (or 'gaps') in the complement correspond to pieces of the gradient-like part.

To give a somewhat more precise description of our results, let us assume that $\rho(f)$ has finitely many components and thus there are a finite number of complementary gaps. To each component $c$ of $\rho(f)$ there corresponds an invariant set $\Lambda(c)$ defined as the set of points whose forward and backward rotation sets lie in $c$. To each gap $J$ there corresponds a homotopically non-trivial circle $\Gamma(J)$ with $f(\Gamma(J)) \cap \Gamma(J)=\varnothing$. Moreover, this correspondence is monotone in the sense that if $c$ lies between two gaps $J_{1}$ and $J_{2}$, then $\Lambda(c)$ is in the annulus bounded by $\Gamma\left(J_{1}\right)$ and $\Gamma\left(J_{2}\right)$. The existence of the $\Gamma(J)$ allows one to think of the positive tilt map as being built up by gluing together finitely many twist maps, each with a connected rotation set. In addition, since for each $\Gamma(J), f(\Gamma(J)) \cap \Gamma(J)=\varnothing$, under iteration each $\Gamma(J)$ must go above or below itself, and this creates an annular gap across which orbits can only transit in one direction. This allows us to assign an orientation to the gaps of $\rho(f)$ which, in turn, induces a partial order on the components of $\rho(f)$ and thus on the collection of the $\Lambda(c)$. This collection with the induced order is a Morse decomposition.

We note that Le Calvez [27] and Casdagli [13] have studied dissipative twist maps and there is some overlap between their techniques and ours. The common heritage of many of these ideas is Birkhoff ([6], [8], cf. [14]). Bernstein [5] studies twist maps with the graph intersection property. We also note that Chenciner in his ongoing study of degenerate Hopf bifurcations has employed many of these same ideas (see [33] for an exposition) and that twist maps such as we study sometimes occur as the time $T$ map of periodically forced oscillations (for example, see [25]).

We now outline the paper. In $\S 1$ we state some results of Franks from [19]. A main ingredient in Franks' paper is an implication he draws from Brouwer's characterization of fixed point free orientation-preserving homeomorphisms of the plane, namely, that such maps cannot have chain recurrent points. If $f$ is a homeomorphism of the annulus with the appropriate properties, by applying this result, he obtains fixed points of various lifts of powers of $f$ and thus periodic orbits with various rotation numbers for $f$.

We adopt this point of view in $\$ 2$ where we characterize fixed point free lifts of positive tilt maps. Just as above, this allows us, under the appropriate hypothesis on $f$, to prove the existence of periodic orbits with various rotation numbers. Because we are working with positive tilt maps, a result of Hall ([20], [21]) allows us to then infer the existence of periodic orbits which are nicely ordered around the annulus under iteration. Then, by taking Hausdorff limits as in Katok ([26], cf. [18] and [3]), one obtains nice minimal sets with irrational rotation numbers. 
In addition to being useful in proving the existence of periodic orbits, the characterization of fixed point free lifts of positive tilt maps is also useful in that it shows that such a map always has an attractor-repeller pair (in the sense of Conley) which is closely related to the asymptotic behaviour of orbits as measured by the rotation number. This is the main tool used to develop the correspondence of $\rho(f)$ to Morse decompositions which is given in $\S 3$.

Section 4 contains some applications of the previous results. The first is a number of alternative hypotheses under which the conclusions of the Aubry-Mather theorem hold. The second application involves the notion of the rotation band of an invariant set that was studied in two previous papers $([12],[11])$. There we showed that an area-preserving monotone twist map never has invariant circles whose rotation numbers lie in the interior of the rotation band of an invariant set with a dense orbit. Here we show that for a (not necessarily area-preserving) positive tilt map, for each number in the rotation band of a chain transitive set, one always has an Aubry-Mather minimal set with that rotation number. Thus, for example, the existence of a 'badly ordered' periodic orbit in a twist map implies the existence of an interval of rotation numbers for the map (as well as positive entropy; see [10]). This last result was one of the main motivations for this paper. If one could show that the badly ordered periodic orbits in the standard circle map family continue up a dimension into orbits for the dissipative standard family in the annulus, then this result could be utilized to construct the bifurcation diagram of the annulus family using the diagram of the circle family given in [9].

\section{Some results of Franks}

We begin with some basic definitions. Let $S^{1}=\mathbf{R} / Z, A=S^{1} \times[0,1], \tilde{A}=\mathbf{R} \times[0,1]$, $T: \tilde{A} \rightarrow \tilde{A}$ be the deck transformation $T(z)=z+(1,0)$, and $\pi_{1}: \tilde{A} \rightarrow \mathbf{R}$ and $\pi_{2}: \tilde{A} \rightarrow$ $[0,1]$ be the projections onto the first and second components respectively. The inner and outer boundaries of $A$ are $\partial_{1}=S^{1} \times\{0\}$ and $\partial_{2}=S^{1} \times\{1\}$. We will use the same notation for the lower and upper boundaries of $\tilde{A}$ and so $\partial_{1}=\mathbf{R} \times\{0\}$ and $\partial_{2}=\mathbf{R} \times\{1\}$. Given a set $Z \subseteq A$, we let $\tilde{Z}$ denote its lift to $\tilde{A}$ and conversely, if $\tilde{Z} \subseteq \tilde{A}$, its projection to $A$ is denoted $Z$.

If $f: A \rightarrow A$ is a homeomorphism isotopic to the identity and $z \in A$, then the rotation set of $z, \rho(z, f)$ is the closed interval

$$
\rho(z, f)=\left[\liminf _{n \rightarrow \infty} \frac{\pi_{1}\left(F^{n}(\tilde{z})-\pi_{1}(\tilde{z})\right)}{n}, \limsup _{n \rightarrow \infty} \frac{\pi_{1}\left(F^{n}(\tilde{z})-\pi_{1}(\tilde{z})\right)}{n}\right],
$$

where $F: \tilde{A} \rightarrow \tilde{A}$ is the lift of $f$ satisfying $F(0,0) \subseteq[0,1) \times\{0\}$. The backwards rotation set of $z$ is defined as $\rho^{*}(z, f)=-\rho\left(z, f^{-1}\right)$. Given an invariant set $Z \subseteq A$, the rotation set of $Z$ is $\rho(Z)=\bigcup\{\rho(z, f): z \in Z\}$ and the rotation set of $f$ is $\rho(f)=\rho(A)$. If $G: \tilde{A} \rightarrow \tilde{A}$ is a homeomorphism satisfying $G(z+(1,0))=G(z)+(1,0)$, we define $\rho(\tilde{z}, G)$ using the liminf and limsup as above.

Given a set $X$ in a topological space $M$, the notations $\mathrm{Cl}(X), \operatorname{Int}(X), \operatorname{Fr}(X)$ and $X^{\mathbf{c}}$ denote the closure, interior, frontier and complement of $X$ respectively. If $f: M \rightarrow M$ is a homeomorphism, then the $\alpha$ - and $\omega$-limit sets of $X$ under $f$ are the 
largest invariant sets of $f$ contained in $\mathrm{Cl}\left(\bigcup_{i=0}^{\infty} f^{-i}(X)\right)$ and $\mathrm{Cl}\left(\bigcup_{i=0}^{\infty} f^{i}(X)\right)$ respectively and are denoted $\alpha(X, f)$ and $\omega(X, f)$ (we will often omit the dependence on $f)$. The $\alpha$ - and $\omega$-limit sets are always closed in $M$. Finally, if $X \subseteq R$, then $\langle X\rangle$ denotes the convex hull of $X$ in $\mathbf{R}$, and if $p, q \in \mathbf{R}, q \cdot X-p=\{q x-p: x \in X\}$.

Our first lemma gives some elementary properties of these notions. The proof of (a) is similar to several in [2], while that of (b) follows the proof of corollary 2.5 in [19] rather closely.

LEMMA 1.1. Let $f: A \rightarrow A$ be a homeomorphism isotopic to the identity.

(a) If $F$ is a lift of $f$ and $z \in \tilde{A}$, then $\rho\left(z, T^{-p} F^{q}\right)=q \cdot \rho(z, F)-p$.

(b) For all $x \in A, \rho(x) \subseteq\langle\rho(\omega(x))\rangle$.

Proof. Since it is obvious that $\rho\left(z, T^{-p} F\right)=\rho(z, F)-p$, we only show $\rho\left(z, F^{q}\right)=$ $q \rho(z, F)$. Let $\left\{n_{i}\right\}$ be a sequence with

$$
\frac{\pi_{1}\left(F^{n_{i}}(x)\right)}{n_{i}} \rightarrow \liminf _{n \rightarrow \infty}\left(\frac{\pi_{1}\left(F^{n}(x)\right)}{n}\right)
$$

and choose $r_{i}$ with $0 \leq r_{i}<q$ and $n_{i}=q m_{i}+r_{i}$ for some $m_{i} \in \mathbf{N}$. Using the compactness of $A$ and the continuity of $f$, there is a constant $K$ so that $\left|\pi_{1}\left(F^{r_{i}}(y)\right)-\pi_{1}(y)\right|<K$ for all $y \in A$ and all $r_{i}$. This implies that

$$
\left|\frac{\pi_{1}\left(F^{n_{i}}(x)\right)}{n_{i}}-\frac{\pi_{1}\left(F^{m_{i} q}(x)\right)}{m_{i} q}\right| \rightarrow 0
$$

and so

$$
\liminf _{n \rightarrow \infty} \frac{\pi_{1}\left(F^{n}(x)\right)}{n} \geq \frac{1}{q}\left(\liminf _{n \rightarrow \infty} \frac{F^{n q}(x)}{n}\right) .
$$

The reverse inequality is obvious and so $q \cdot \inf (\rho(z))=\inf \left(\rho\left(z, F^{q}\right)\right)$. The proof for the supremum is similar, which proves (a).

Assume (b) is false, and so inf $(\rho(x))<\inf (\rho(\omega(x)))$ or $\sup (\rho(x))>$ $\sup (\rho(\omega(x)))$. We assume the former; the proof assuming the latter is similar. Pick a $p / q$ with inf $(\rho(x))<p / q<\inf (\rho(\omega(x)))$ and let $G=T^{-p} F^{q}$. Using (a) and the fact that $\omega\left(x, f^{q}\right) \subseteq \omega(x, f)$, we have inf $\rho(x, G)<0<\inf \rho(\omega(x, g), G)$. Since $\omega(x, g)$ is compact, we may find an $N$ with $\pi_{1}\left(G^{N}(z)\right)-\pi_{1}(z)>2$ for all $z \in \tilde{\omega}(x, g)$. Since $g^{i}(x)$ converges to the compact set $\omega(x, g)$, for $n$ sufficiently large, $\pi_{1}\left(G^{N}\left(G^{k N+n}(x)\right)\right)-\pi_{1}\left(G^{k N+n}(x)\right)>1$ for all $k \in N$. Using this inequality for $k=0$, $1, \ldots, k$ and adding, we get

$$
\pi_{1}\left(G^{k N}\left(G^{n}(x)\right)\right)-\pi_{1}\left(G^{n}(x)\right)>k
$$

and so $\rho\left(g^{n}(x), G^{N}\right) \subseteq[1, \infty)$ and so $\rho(x, G)=\rho\left(q^{n}(x), G^{N}\right) / N \subseteq[1 / N, \infty)$, a contradiction.

The following example shows that the convex hull is needed in (b) of this lemma, i.e. $\rho(x) \subseteq \rho(\omega(x))$ is false in general. We give an example in the two shift. It can be transferred to the annulus via a semiconjugacy as in [25]. The asymptotic average of the number of ones in a sequence gives the rotation number of the corresponding 
point in the annulus. Let $s$ be a sequence consisting of alternating blocks of all ones or all zeros with the blocks increasing in length. By adjusting the length of the blocks, we can make the rotation number of the sequence equal to any number in $[0,1]$. However, $\omega(x)$ is precisely sequences of the following four types: infinitely many zeros followed by infinitely many ones, infinitely many ones followed by infinitely many zeros, all ones, or all zeros. Thus $\rho(\omega(s))=\{0,1\}$.

Our next theorem is a consequence of results of Franks [19]. Given our definition of $\rho(f)$, it is not clear that $r \in \rho(f)$ implies the existence of a point $z$ whose rotation number equals $r$ in the 'traditional sense', i.e. $\rho(z)=r$ and not just $r \in \rho(z)$. Franks shows that this actually is the case if $f$ is a homeomorphism and $r=p / q$, a rational. In fact, one may choose $z$ to be a periodic orbit with least period $q$. (Note: here and throughout this paper, if we write $p / q$ we are assuming $(p, q)=1$.) Such an orbit is called a $p / q$-periodic orbit and is clearly the simplest type of orbit with rotation number $p / q$. The analogous result for the case when $r$ is irrational is known when $f$ is positive tilt (see theorem 2.2) and appears to be unknown when $f$ is a homeomorphism.

The second part of theorem 1.2 concerns the rotation sets of chain transitive sets. The finite sequence $x=x_{0}, x_{1}, \ldots, x_{n}=y$ is called an $\varepsilon$-chain from $x$ to $y$ if for all $i, d\left(f\left(x_{i}\right), x_{i+1}\right)<\varepsilon$. A point $x$ is chain recurrent if for all $\varepsilon>0$ there exists an $\varepsilon$-chain from $x$ to $x$. A set $Z$ is chain transitive if for all $\varepsilon>0$ and all $x, y, \in Z$ there exists an $\varepsilon$-chain from $x$ to $y$. The $\alpha$ - and $\omega$-limit sets of a point are examples of chain transitive sets. Theorem $1.2(b)$ says that each rational $p / q$ in the convex hull of $\rho(Z)$ is in the rotation set and so $f$ has a $p / q$-periodic orbit by $1.2(a)$. Note that even though $Z$ is chain transitive, $\rho(z)$ could be properly contained in $\langle\rho(z)\rangle$ and so, in particular, the $p / q$-periodic orbit may not be in $Z$. A generalization of this result to positive tilt maps is given in theorem $4.1(\mathrm{~b})$.

THEOREM 1.2 (Franks). Let $f: A \rightarrow A$ be a homeomorphism isotopic to the identity.

(a) For all $p / q \in \rho(f)$, $f$ has a $p / q$-periodic orbit.

(b) If $Z$ is a compact chain transitive set, then $\langle\rho(Z)\rangle \cap Q \subseteq \rho(f)$.

\section{Fixed point free lifts of positive tilt maps}

For the balance of the paper we will be focusing on positive tilt maps of the annulus, which we define shortly. The image of a smooth embedding $\gamma:[0,1] \rightarrow \tilde{A}$ is called a boundary-connecting arc if $\gamma(0) \in \partial_{1}, \gamma(1) \in \partial_{2}$ and $\gamma(0,1) \subseteq \operatorname{Int}(\tilde{A})$. Since $\gamma$ is smooth, we may define a continuous $\theta(t)$ which measures the angle between $\gamma^{\prime}(t)$ and the vertical and satisfies $-\pi \leq \theta(0) \leq \pi$. If $\theta<0$, then $\gamma$ is called a positively tilted boundary-connecting arc (see figure 1). An arc of the form $r \times[0,1]$ for some $r \in \mathbf{R}$ is called a vertical arc. Given a $z \in \tilde{A}$, the vertical arc containing $z$ will be denoted $I_{z}$. We let $I_{z}^{+}=\left\{p \in I_{z}: \pi_{2}(p)>\pi_{2}(z)\right\}$ and $I_{z}^{-}=\left\{p \in I_{z}: \pi_{2}(p)<\pi_{2}(z)\right\}$. If $I$ is a vertical arc and $J$ a boundary-connecting arc with $I \cap J \neq \varnothing$, we let top $(I \cap J)$ and bot $(I \cap J)$ denote the highest and lowest points on $I \cap J$ respectively (see figure 1).

If $F: \tilde{A} \rightarrow \tilde{A}$ is a diffeomorphism that satisfies $F(z+(1,0))=F(z)+(1,0)$, and if for all $z \in \tilde{A}, F\left(I_{z}\right)$ is a positively tilted arc, we call $F$ a positive tilt map. If $f: A \rightarrow A$ 


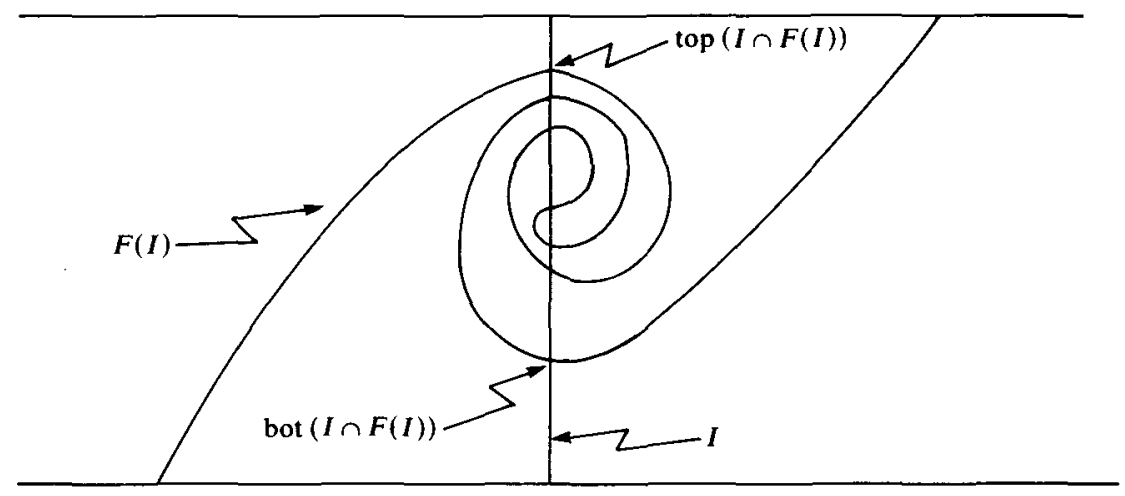

Figure 1

is the projection of a positive tilt map $F$, then $f$ is also termed a positive tilt map. The set of all such $f$ with the $C^{1}$-topology will be denoted $P T$. Note that $P T$ is an open subset of $\operatorname{Diff}_{1}(A)$. If a positive tilt map $F$ has the property that for all $x$, $z \in \tilde{A}, F\left(I_{z}\right) \cap I_{x}$ contains at most one point, then $F$ is called monotone twist. Note that if $F$ is monotone twist, $F^{q}$ may not be monotone twist, but it will be positive tilt because $P T$ is closed under iteration. One last definition: a point $z$ which satisfies $F(z) \in I_{z}$ is called an up point if $\pi_{2}(F(z))>\pi_{2}(z)$ and a down point if $\pi_{2}(F(z))<$ $\pi_{2}(z)$.

The next lemma gives some elementary properties of positive tilt maps that essentially go back to Birkhoff ([6], [7] and [8]). For an exposition of Birkhoff's work see [24, Ch. 1].

LEMMA 2.1. (a) If $\gamma:[0,1] \rightarrow A$ is a positively tilted boundary-connecting arc, then $\pi_{1}(\gamma(0))<\pi_{1}(t)<\pi_{1}(\gamma(1))$ for all $t \in(0,1)$. In addition, if $I$ is any vertical arc with $\gamma \cap I \neq \varnothing$ and $t_{1}=\min \{t: \gamma(t) \in I\}$ while $t_{2}=\max \{t: \gamma(t) \in I\}$, then $\operatorname{top}(\gamma \cap I)=$ $\gamma\left(t_{1}\right)$ and bot $(\gamma \cap I)=\gamma\left(t_{2}\right)$.

(b) Let $F: \tilde{A} \rightarrow \tilde{A}$ be a positive tilt map.

(i) If $I$ is a vertical arc with $I \cap F(I) \neq \varnothing$, then $F\left(\right.$ bot $\left.\left(F^{-1}(I) \cap I\right)\right)=$ top $(I \cap F(I))$ and $F$ (top $\left.\left(F^{-1}(I) \cap I\right)\right)=$ bot $(I \cap F(I))$.

(ii) If $z$ is a down point for $F$ and $I$ is a vertical arc containing $z$, then top $\left(F^{-1}(I) \cap I\right)$ is also a down point. If $z$ is an up point, then so is bot $\left(F^{-1}(I) \cap I\right)$.

Proof. For (a) see [24, p. 21].

The fact in (b)(i) follows from (a). To prove (b)(ii), note that since $z$ is a down point, then $z \in F^{-1}(I) \cap I$ and $F(z) \in I \cap F(I)$ and so

$$
\begin{gathered}
\pi_{2}\left(\operatorname{top}\left(F^{-1}(I) \cap I\right)\right) \geq \pi_{2}(z)>\pi_{2}(F(z)) \geq \pi_{2}(\text { bot }(F(I) \cap I)) \\
=\pi_{2}\left(F\left(\operatorname{top}\left(F^{-1}(I) \cap I\right)\right)\right) \text { using }(\mathrm{b})(\mathrm{i}) .
\end{gathered}
$$

If we let twist $(f)=\left[\rho\left(\partial_{1}\right), \rho\left(\partial_{2}\right)\right]$, then the first sentence of (a) above implies that $\rho(f) \subseteq$ twist $(f)$ when $f \in P T$. The next theorem answers the question raised after lemma 1.1 for positive tilt $f$. For any $r \in \rho(f)$ there always exists a 'nice' minimal 
set $Z$ with $\rho(Z)=r$. If $r=p / q$, then the minimal set is a $p / q$-periodic orbit as in theorem 1.2, and if $r$ is irrational, $Z$ is now an invariant circle or a Denjoy minimal set. In addition, these minimal sets are 'nicely ordered' around the annulus under iteration.

These results in the context of area-preserving monotone twist maps were first obtained by Aubry and Mather. Katok [26] (see also [18]) gives an alternative proof of the Aubry-Mather theorem using the technique of taking Hausdorff limits of periodic orbits to obtain 'quasi-periodic' orbits (circles or Denjoy minimal sets). From our point of view, the advantage of this approach is that it only depends on the monotone twist (or positive tilt) hypothesis and not on the fact that $f$ is area-preserving.

We now define the notion of a 'nicely ordered' invariant set for a positive tilt map. In this we are following Hall [21], who generalizes these notions from their application to monotone twist maps. Our presentation follows that of Katok [26].

An $f$-invariant set $Z \subseteq A$ is called monotone if

(1) $\pi_{1}$ restricted to $\tilde{Z}$ is injective;

(2) $F$ acting on $\tilde{Z}$ is order-preserving in the sense that if $z_{1}, z_{2} \in \tilde{Z}$ and $\pi_{1}\left(z_{1}\right)<$ $\pi_{2}\left(z_{2}\right)$, then $\pi_{1}\left(F\left(z_{1}\right)\right)<\pi_{1}\left(F\left(z_{2}\right)\right)$.

If, in addition,

(3) for each $z_{1}, z_{2} \in \tilde{Z}$, any intersection of $I_{F\left(z_{1}\right)}^{-}$and $F\left(I_{z_{2}}^{+}\right)$can be removed by isotopy rel $F\left(z_{1}\right), F\left(z_{2}\right), \partial_{1}$ and $\partial_{2}$, then $Z$ is called link-monotone for $f$.

For a monotone twist map (1) and (2) imply (3), but if $F$ is positive tilt, $z_{1}$ and $z_{2}$ could wrap all the way around each other under $F$ and still satisfy (2) as in figure 2. This means that we must require (1), (2) and (3) for a positive tilt map in order to insure that the collection of such sets will be closed under Hausdorff limits.

An invariant set that is both link-monotone and minimal will be called an Aubry-Mather set. If $Z$ is monotone, then the action of $f$ on $Z$ can be extended to a circle homeomorphism. This implies that if $Z$ is monotone, $\rho(Z)=\rho^{*}(Z)$ and is a single number. It also implies that if $Z$ is Aubry-Mather, then $\rho(Z)=p / q$ implies

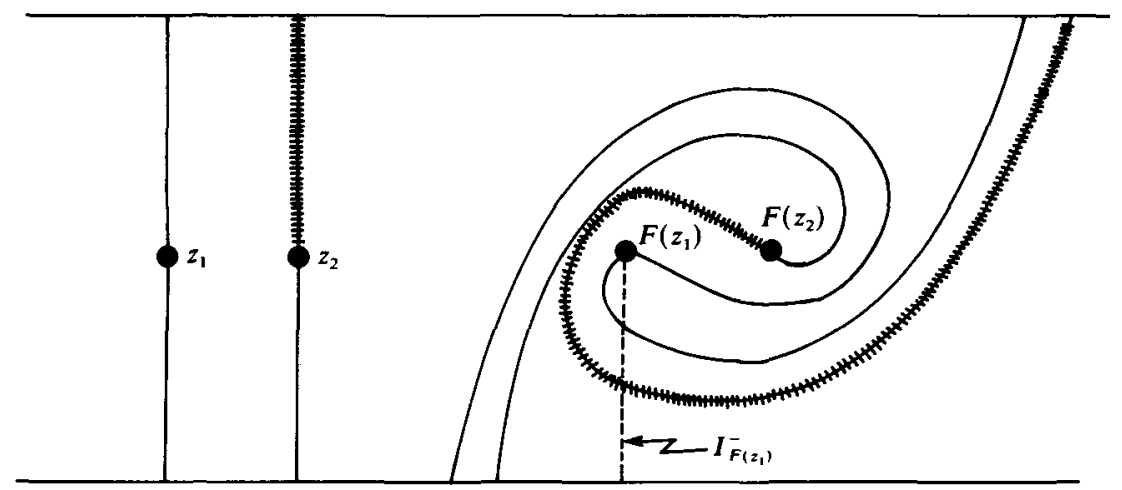

Figure 2 
that $Z$ is a $p / q$-periodic orbit (sometimes called a Birkhoff periodic orbit), and if $\rho(Z) \notin \mathbf{Q}$, then $Z$ is a circle or a Denjoy minimal set. If $Z$ is link-monotone, then $\mathrm{Cl}(Z)$ is also, so whenever $f$ has a link-monotone set with a given rotation number, it also has an Aubry-Mather one. We shall also need the following property of link-monotone sets whose proof is similar to that given in [26] for the monotone twist case. If $F$ is positive tilt, then there exists a Lipschitz constant $c$ so that for any link-monotone set $Z$ for $f$, we may find a Lipschitz $\phi: \mathbf{R} \rightarrow[0,1]$ with Lipschitz constant $c$ so that $\tilde{Z} \subseteq$ graph $(\phi)$.

If $f \in P T$, define $\rho_{\text {mon }}(f)=\{\rho(Z): Z$ is link-monotone for $f\}$. The next theorem states that this comprises all of $\rho(f)$, i.e. any $r \in \rho(f)$ is represented by an AubryMather set with rotation number $r$. Note that this result is basically just a combination of results from [19], [21] and [26], and the basic ideas have been utilized in [27], [13] and elsewhere.

THEOREM 2.2. If $f \in P T$, then $\rho(f)=\rho_{\text {mon }}(f)$ and is a closed set. In particular, if $p / q \in \rho(f)$, then $f$ has a Birkhoff $p / q$-periodic orbit and for each irrational $\alpha \in \rho(f)$, $f$ has an Aubry-Mather Denjoy minimal set or an invariant circle with rotation number $\alpha$.

Proof. The proof rests on two observations. The first is that $\left\{\left(f, \rho_{\operatorname{mon}}(f)\right): f \in P T\right\}$ is closed in $P T \times \mathbf{R}$. In [26] Katok shows that for fixed $f \in P T, \rho_{\text {mon }}(f)$ is closed. Our proof of the first observation follows his closely. We indicate the minor alterations needed when one allows the function to vary.

Let $f_{k} \rightarrow f_{0}$ in $P T$ and suppose $r_{k} \in \rho_{\text {mon }}\left(f_{k}\right)$ and $r_{k} \rightarrow r_{0}$. For each $k$ choose $\Sigma_{k}$ a link-monotone set for $f_{k}$ with $\rho\left(\Sigma_{k}\right)=r_{k}$. As noted above, for each $k$ we may find a $c_{k}>0$ which is a Lipschitz constant for link-monotone sets of $f_{k}$. By virtue of the $C^{1}$-convergence $f_{k} \rightarrow f_{0}$, the $c_{k}$ may be chosen so that lim sup $\left\{c_{k}\right\}$ will be finite and any $c_{0}$ larger than it will be a Lipschitz constant for link-monotone sets for $f_{0}$. This insures that any $\Sigma_{0}$ which is a limit point of $\left\{\Sigma_{k}\right\}$ in the Hausdorff topology is a link-monotone set for $f_{0}$ with $\rho\left(\Sigma_{0}\right)=r_{0}$. Such a $\Sigma_{0}$ must exist because the set of closed subsets of $\boldsymbol{A}$ with the Hausdorff topology is compact.

The second observation is that for $f \in P T, p / q \in \rho(f)$ implies that $p / q \in \rho_{\text {mon }}(f)$. This is a straightforward consequence of Franks' theorem (theorem 1.2), which shows that $p / q \in \rho(f)$ implies that $f$ has a $p / q$-periodic orbit, and a theorem of Hall [21] which says that any time a positive tilt map has a $p / q$-periodic orbit it has a link-monotone $p / q$-periodic orbit.

Now by the first observation, $\rho_{\text {mon }}(f)$ is a closed set. In the light of the second observation, to show that $\rho_{\text {mon }}(f)=\rho(f)$, we need only show that if $\alpha \in \rho(f)$ and $\alpha \notin \mathrm{Cl}(\rho(f) \cap Q)$, then $\alpha \in \rho_{\text {mon }}(f)$. To prove this, choose an $x \in A$ with $\alpha \in \rho(x, f)$. Now by lemma 1.1(b) we have $\alpha \in \rho(x) \subseteq\langle\rho(\omega(x))\rangle$. Since $\omega(x)$ is chain transitive, Franks' theorem and our assumptions on $\alpha$ imply that $\rho(\omega(x))=\alpha$. To show then that $\alpha \in \rho_{\text {mon }}(f)$, we use the $C^{1}$-closing lemma to perturb $f$ to $f_{k}$, where $f_{k}$ has a $p_{k} / q_{k}$-periodic orbit with $p_{k} / q_{k} \rightarrow \alpha$. We then use our two observations to conclude that $\alpha \in \rho_{\text {mon }}(f)$. We postpone the details till after theorem 4.1, as we need a result proved there independently of this result. 
We remark that since for an Aubry-Mather set $\Sigma, \rho(\Sigma)=\rho^{*}(\Sigma)$ and is a single number, theorem 2.2 implies that $\rho(f)=\rho^{*}(f)$. The next theorem gives our characterization of fixed point free positive tilt maps. Before stating it, we give an example that will help clarify its contents. We shall need the example later, so we state it in a somewhat more general form than is necessary now.

Example 2.1. Define $S, T:[a, b] \times \mathbf{R} \rightarrow[a, b] \times \mathbf{R}$ via $S(x, y)=(x+y, y)$ and $T(x, y)=$ $(r, \phi(y))$, where $\phi:[a, b] \rightarrow[a, b]$ is a diffeomorphism with $\phi(a)=a$ and $\phi(b)=b$. If $G=S \circ T$, then $G$ is a monotone twist map.

Now let $[a, b]=[-1,1]$ and choose $\phi_{1}$ and $\phi_{2}$ as above but with $\phi_{1}(x)>x$ and $\phi_{2}(x)<x$ for $x \in(a, b)$, and for $i=1,2$ let $T_{i}(x, y)=\left(x, \phi_{i}(y)\right)$ and $G_{i}=S \circ T_{i}$. Both $G_{1}$ and $G_{2}$ will be fixed point free monotone twist maps whose orbits will move along the lines shown in figures $3(a)$ and (b) respectively. Note that this is essentially the same example given in [19] as a fixed point free homeomorphism of $\tilde{A}$.

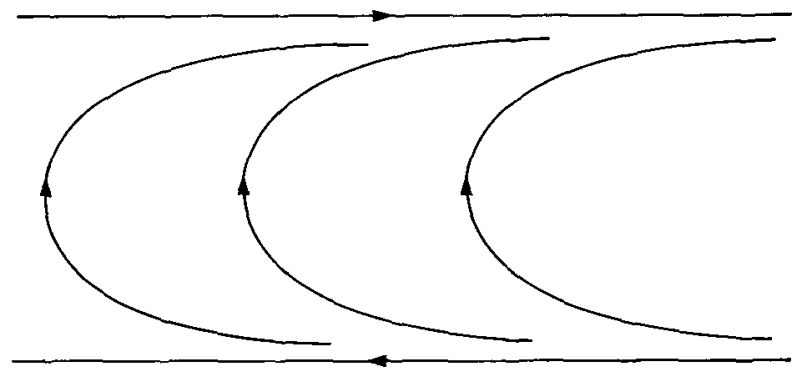

(a)

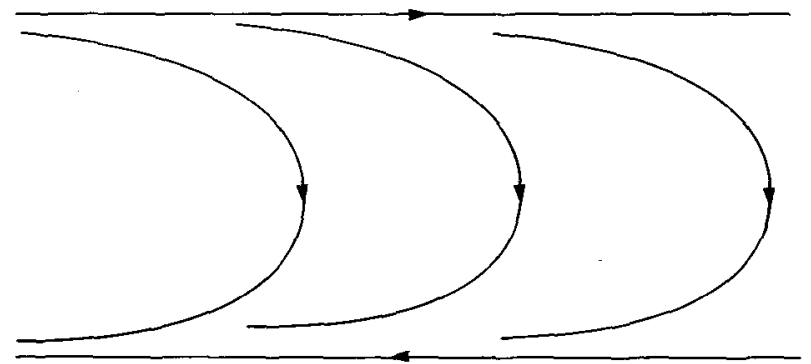

(b)

Figure 3

In a certain sense $G_{1}$ and $G_{2}$ are the only examples of fixed point free positive tilt maps. More precisely, the features they share with any other fixed point free positive tilt map are as follows. (1) There are three categories of orbits: those that always move left, those that always move right and those that change direction. Moreover, those that change direction only do so once and all these orbits do so in the same way (left to right or right to left). (2) The orbits that always go left or always go right are the limit sets of those that change direction. (3) There is a homotopically non-trivial curve that 'separates' orbits going left from those going right. What distinguishes $G_{1}$ from $G_{2}$ is whether the orbits which change direction 
are going upward or downward. This motivates the following definition. A positive tilt $F$ is called an up map if it has an up point and a down map if it has a down point.

Our theorem will be stated in terms of sets defined as follows. Given $F$, define $\Delta=\left(\Delta_{1}, \Delta_{2}\right): A \rightarrow \mathbf{R}^{2}$ via $\Delta_{i}(\tilde{z})=\pi_{i}(F(\tilde{z}))-\pi_{i}(\tilde{z})$ for $i=1$ or 2 . Let

$$
\begin{gathered}
R(F)=\left\{\tilde{z}: \Delta_{1}(\tilde{z})>0\right\}, \quad L(F)=\left\{\tilde{z}: \Delta_{1}(\tilde{z})<0\right\}, \quad V(F)=\left\{\tilde{z}: \Delta_{1}(\tilde{z})=0\right\} \\
R^{\infty}(F)=\{\tilde{z}: o(\tilde{z}) \subseteq R(F)\} \quad \text { and } \quad L^{\infty}(F)=\{z: o(\tilde{z}) \subseteq L(F)\}
\end{gathered}
$$

where $o(\tilde{z})=\left\{\ldots, F^{-1}(\tilde{z}), \tilde{z}, F(\tilde{z}), \ldots\right\}$ is the orbit of $\tilde{z}$ under $F$. We will often delete the dependence of these sets on $F$. We use the same notation for the projection of these sets into $A$.

For our examples $G_{1}$ and $G_{2}$ above, $R^{\infty}=\partial_{2}, L^{\infty}=\partial_{1}$ and $V$ is the horizontal line $y=\phi_{i}^{-1}(0)$. The difference between these simple examples and a more general fixed point free positive tilt map lies primarily in the complication of the dynamics with $L^{\infty}$ and $R^{\infty}$.

We shall also need some notions from Conley ([16] and [17]). Conley worked with flows; we adapt the notions to homeomorphisms and leave the adaptation of any required proofs to the reader. If $f: M \rightarrow M$ is a homeomorphism, then $X \subseteq M$ is an attractor (or attracting set) if for some neighbourhood $N$ of $X, X=\omega(N)$. Note that this definition contains no requirement of indecomposibility or transitivity in $X$. The set $X$ is called a repeller if it is an attractor for $f^{-1}$. If $X$ is an attractor, one can show that $X^{*}=\{z \in M: \omega(z) \cap X=\varnothing\}$ is a repeller which is called the complementary repeller to $X$, and $\left(X, X^{*}\right)$ are called an attractor-repeller pair. The set $C\left(X, X^{*}\right)$ is defined to be $M-\left\{X \cup X^{*}\right\}$ and consists of the connecting orbits of the attractor-repeller pair. This name is appropriate since $C\left(X, X^{*}\right)=$ $\left\{z \in M: \alpha(z) \subseteq X^{*}\right.$ and $\left.\omega(z) \subseteq X\right\}$. If $N$ is a set with $f(\mathrm{Cl}(N)) \subseteq \operatorname{Int}(N)$, then $\bigcap_{i=0}^{\infty} f^{i}(N)$ is an attractor.

Our last remark before the theorem is a straightforward consequence of well known plane topology (e.g. the Riemann mapping theorem). If $U \subseteq A$ is a connected open set such that $U \cap \partial_{i}=\varnothing$ for $i=1,2$, and each simple closed curve $\Gamma \subseteq U$ that is null homotopic in $A$ is contractible within $U$, and $U$ contains a simple closed curve that is homotopically non-trivial in $A$, then $U$ is homeomorphic to an open annulus.

The main part of the theorem (part (b)) is stated for a fixed point free down map. There is, of course, an analogous result for up maps. We also note that part (a) is essentially the fixed point theorem used by Hall in [20], [21] and [12].

Theorem 2.3. Let $F: \tilde{A} \rightarrow \tilde{A}$ be a positive tilt map with $0 \in$ twist $(F)$.

(a) If $F$ is an up map and a down map, then $F$ has at least two fixed points. If $F$ is neither an up map nor a down map, then $F$ has a fixed point.

(b) If $F$ is a fixed point free down map, then $L^{\infty}$ and $R^{\infty}$ are an attractor-repeller pair for f. In addition, $L^{\infty}$ and $R^{\infty}$ are each connected and $C\left(R^{\infty}, L^{\infty}\right)$ is homeomorphic to an open annulus and is homotopically non-trivial in $A$. Given $z \in C\left(R^{\infty}, L^{\infty}\right)$, there exists a $k \in \mathbf{Z}$ with $f^{i}(z) \in R$ for $i<k, f^{k}(z) \in L \cup V$ and $f^{i}(z) \in L$ for $i>k$. In particular, $C\left(R^{\infty}, L^{\infty}\right)=\left\{z \in A: \rho(z) \subseteq\left(0, \rho\left(\partial_{2}\right)\right]\right.$ and $\left.\rho^{*}(z) \subseteq\left[\rho\left(\partial_{1}\right), 0\right)\right\}$. 
Proof. We prove the second statement of (a) first. Since $0 \in$ twist $(F), \Delta_{1}\left(\partial_{1}\right) \leq 0$ and $\Delta_{1}\left(\partial_{2}\right) \geq 0$, and since $\Delta(\tilde{A})$ is connected, it must intersect the $y$-axis. However, since $F$ is neither up nor down, this intersection must be at the origin and any $z \in \Delta^{-1}(0,0)$ is a fixed point.

As noted above the theorem, the first sentence of (a) is essentially the fixed point theorem used by Hall. We include a sketch of its proof for the sake of completeness. By lemma 2.1(b)(ii), we may find an up point $u$ and a down point $d$ with $d=\operatorname{top}\left(F^{-1}\left(I_{d}\right) \cap I_{d}\right)$ and $u=\operatorname{bot}\left(F^{-1}\left(I_{u}\right) \cap I_{u}\right)$. Translating these by an integer if necessary, we may assume that $\pi_{1}\left(F^{-1}\left(I_{d} \cap \partial_{1}\right)\right)<\pi_{1}(u)$ and $\pi_{1}(d)<$ $\pi_{1}\left(F^{-1}\left(I_{u} \cap \partial_{2}\right)\right)$. These choices of $u$ and $d$ along with lemma 2.1 ensure that the situation depicted in figure 4 is accurate. In particular, the box depicted in figure 4(a) actually has an embedded circle for a boundary and its image looks like figure 4(b). In particular, for $z$ an element of the dotted portion of the boundary, $\Delta_{1}(z)<0$, and for $z$ in the solid portion, $\Delta_{1}(z)>0$. Now since $\Delta_{1}(u)=\Delta_{1}(d)=0, \Delta_{2}(u)<0$ and

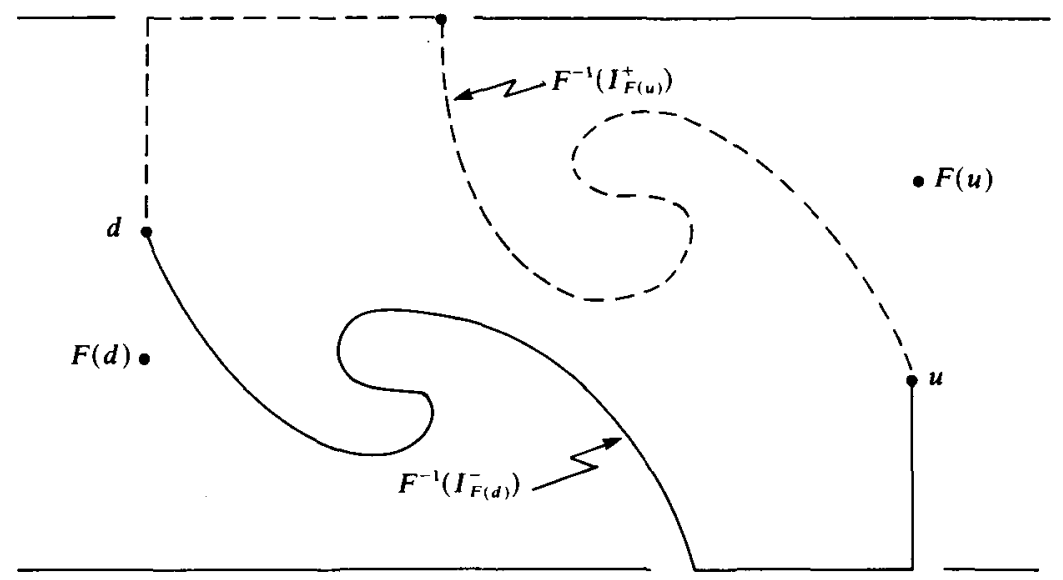

(a)

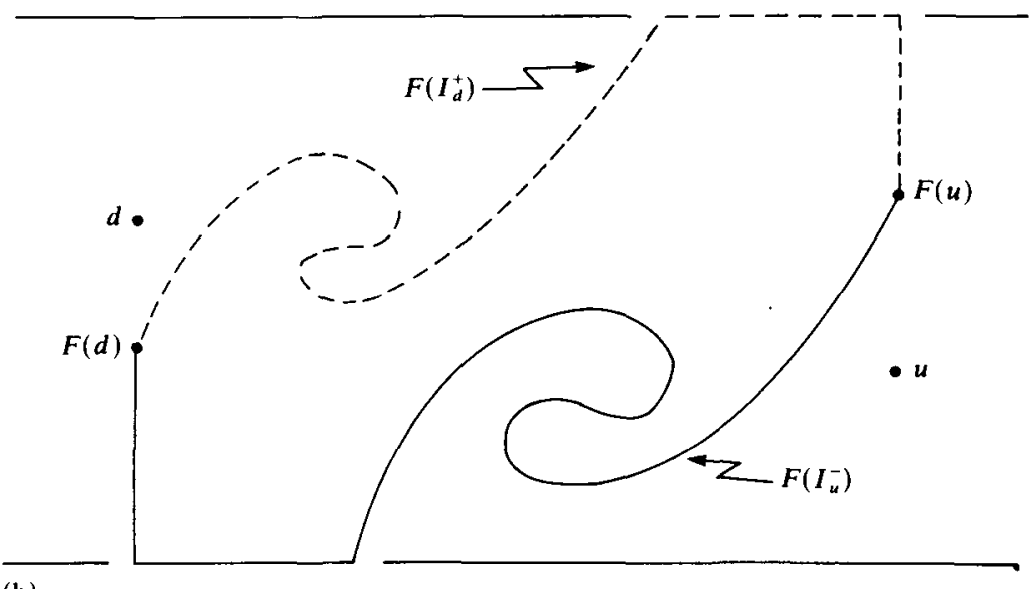

(b)

Figure 4 
$\Delta_{2}(d)>0$, the index of $F$ computed around this box is -1 , so $f$ has at least two fixed points.

Now we assume that $F$ is a fixed point free down map. First we show that for $z \in C\left(R^{\infty}, L^{\infty}\right)$ there exists a $k \in Z$ as in the theorem. If this were not the case, then for some $z, z \in V \cup L$ and $F(z) \in V \cup R$. Let $I=I_{F(z)}$ and $F(z)=\left(x_{1}, y_{1}\right)$. If $z \in V$, then $F(z) \in I \cap F(I)$ and so $y_{1} \leq \pi_{2}(\operatorname{top}(I \cap F(I)))$. If $z \in L$, then $z$ is in the component of $A-I$ that contains positive infinity and so $F(z)$ is in the component of $\tilde{A}-F(I)$ that contains positive infinity. Now if $y_{1}>\pi_{2}(\operatorname{top}(I \cap F(I)))$, this would be impossible so $y_{1} \leq \operatorname{top}(I \cap F(I))$. Similar arguments show that $F(x) \in V \cup R$ implies that bot $\left(F^{-1}(I) \cap I\right) \leq y_{1}$. Thus by lemma $2.1(\mathrm{~b})(\mathrm{i}), F$ has an up point, so using (a) we have a contradiction.

By what we proved in the previous paragraph, $f(V \cup L) \subseteq L$. Since $V \cup L \supseteq \mathrm{Cl}(L)$ and $L$ is open, $\bigcup_{i=0}^{\infty} f^{i}(L)$ is an attracting set which is easily seen to be equal to $L^{\infty}$. The equality $R^{\infty}=\{z: o(z) \cap \mathrm{Cl}(L)=\varnothing\}$ makes it clear that $R^{\infty}$ is the complementary repeller.

To derive the topological properties of $L^{\infty}, R^{\infty}$ and $C\left(L^{\infty}, R^{\infty}\right)$, we find an open annulus $U$ with $L^{\infty}=\bigcap_{i=0}^{\infty} f^{i}(U)$ and $\operatorname{Fr}(U)$ having some nice properties we will need later for lemma 3.2. Define $b: \mathbf{R} \rightarrow[0,1]$ via $b(x)=\pi_{2}\left(\right.$ bot $\left.\left(F^{-1}\left(I_{x}\right) \cap I_{x}\right)\right)$. Since $V=\bigcup_{x \in \mathbf{R}} F^{-1}\left(I_{x}\right) \cap I_{x}$, it is clear that graph $(b) \subseteq V$ (it is its 'lower edge' in the sense to be defined shortly). Now let $U=\{(x, y) \in \tilde{A}: y<b(x)\}$; then $U$ is open and satisfies the properties given above the theorem, which suffice to show that $U-\partial_{1}$ is homeomorphic to an open annulus. Now since $F$ has no up points, lemma 2.1 implies that for all $(x, y) \in V, \pi_{2}(F(x, y))<\pi_{2}(F(x, b(x)))<b(x)$ and so $f(V) \subseteq U$. We claim that this implies that $f(\mathrm{Cl}(L)) \subseteq U$. Since $\operatorname{Fr}(L) \subseteq V$, if this were not the case we could find a $p \in L$ with $F(p) \in U^{c}$. But for any $z \in U^{c}$, by the definition of $U, I_{z}^{+} \subseteq U^{c}$ and thus $I_{F(p)}^{+} \subseteq U^{c}$. But since $F(L) \cap \partial_{2}=\varnothing$, there must be some $p^{\prime} \in I_{F(p)}^{+}$with $p^{\prime} \in \operatorname{Fr}(F(L))=F(\operatorname{Fr}(L)) \subseteq F(V) \subseteq U$, a contradiction.

We have proved the claim, and since $U \subseteq L$,

$$
L^{\infty}=\bigcap_{i=0}^{\infty} f^{i}(L)=\bigcap_{i=0}^{\infty} f^{i}(\mathrm{Cl}(U))
$$

and so $L^{\infty}$ is connected. Since $R^{\infty}$ is the complementary repeller to $L^{\infty}, R^{\infty}=$ $\bigcap_{i=0}^{\infty} f^{-i}\left(U^{c}\right)$ and so $R^{\infty}$ is also connected since $U^{c}$ is. In addition we have $\left(R^{\infty}\right)^{c}=\bigcap_{i=0}^{\infty} f^{-i}(U)$, and since this is an ascending union, $\left(R^{\infty}\right)^{c}-\partial_{1}$ is an open annulus. A similar argument shows that $\left(L^{\infty}\right)^{c}-\partial_{2}$ is an open annulus, and since $\partial_{1} \subseteq L^{\infty}$ and $\partial_{2} \subseteq R^{\infty},\left(L^{\infty}\right)^{c} \cap\left(R^{\infty}\right)^{c}=C\left(L^{\infty}, R^{\infty}\right)$ is also an open annulus. Finally for $z \in C\left(L^{\infty}, R^{\infty}\right)$, the existence of a $k$ with $f^{i}(z) \in L$ for $i>k$ and $f^{i}(z) \in R$ for $i<k$ makes it obvious that $R^{\infty}=\left\{z: \rho(z) \cup \rho^{*}(z) \subseteq\left(0, \rho\left(\partial_{2}\right)\right]\right\}, \quad L^{\infty}=$ $\left\{z: \rho(z) \cup \rho^{*}(z) \subseteq\left[\rho\left(\partial_{1}\right), 0\right)\right\}$ and so

$$
C\left(R^{\infty}, L^{\infty}\right)=\left\{z: \rho^{*}(z) \subseteq\left[\rho\left(\partial_{1}\right), 0\right) \text { and } \rho(z) \subseteq\left(0, \rho\left(\partial_{2}\right)\right]\right\} .
$$

We now note some implications of this result for the sake of comparison with some of the implications Franks draws from Brouwer's characterization of fixed point free plane homeomorphisms in [19]. If an orientation-preserving homeo- 
morphism of the plane has any form of recurrence (e.g. a periodic point or chain recurrent point), then it cannot be fixed point free. Franks uses this to show that if there is a point $z$ with $0 \in \rho(z)$, then $F$ has a fixed point. One implication of theorem 2.3 is that if a positive tilt map $F$ has a point $x$ that goes left, right then left (or right, left then right), then $F$ has a fixed point. This motion of $x$ may be interpreted as a very weak form of recurrent behaviour or as a finite version of the asymptotic statement $0 \in \rho(z)$ used in the homeomorphism case. Franks also shows that if $Z$ is chain transitive for $f$, then one has the trichotomy: every point in $\tilde{Z}$ goes asymptotically to positive infinity, every point of $\tilde{Z}$ goes asymptotically to negative infinity or else $F$ has a fixed point. For positive tilt $F$, one has the trichotomy: every point in $\tilde{Z}$ goes left (under a single iteration), every point in $\tilde{Z}$ goes right or else $F$ has a fixed point. This follows from theorem 2.3 since a chain transitive $Z$ must be in either $L^{\infty}$ or $R^{\infty}$ as they are an attractor-repeller pair. The general theme in these comparisons appears to be that statements which must be checked asymptotically in the homeomorphism case in order to insure the existence of a fixed point need only be checked for a finite number of iterates in the positive tilt case.

We also compare an implication of our theorem with a result of Casdagli [13]. He shows that if $F$ is monotone twist and we let $G=T^{-p} F^{q}$, then any point in $F(V(G)) \cap V(G)$ is a $p / q$-periodic orbit for $f$. It is in general not true that any point in $G(V(G)) \cap V(G)$ is a fixed point for $G$ (and thus a $p / q$-periodic orbit for $f$ ), but theorem 2.3 does imply that if $G(V(G)) \cap V(G) \neq \varnothing$, then $G$ has a fixed point.

\section{Morse decompositions}

In this section we develop the correspondence between Morse decompositions for a positive tilt $f$ and the components of $\rho(f)$. We begin with an informal description of our results which includes a summary of the relevant concepts from Conley ([16], [17], cf. [31]). Our description of Conley's work is, of necessity, brief and somewhat skewed towards our applications. We urge the reader to consult the references for a more balanced and thorough treatment. Also, Conley worked with flows and we work with diffeomorphisms. With a little care all the notions (that we use) can be adapted. Since our description here is informal, we do not take the care needed to distinguish the two types of systems. The necessary concepts will be defined precisely before theorem 3.3 .

Our starting point is Conley's decomposition of a dynamical system $(M, f)$ into the set of chain recurrent points, $R(f)$, and its complement, the set of gradient-like points. The chain recurrent set is further divided into its connected components $\left\{R_{\mu}\right\}$ for $\mu$ in some index set. Since for each $z \in M, \alpha(z)$ and $\omega(z)$ are chain transitive, thus $\alpha(z) \subseteq R_{\mu_{1}}$ and $\omega(z) \subseteq R_{\mu_{2}}$ for some $\mu_{1}$ and $\mu_{2}$. It turns out that $z$ is chain recurrent if and only if $\mu_{1}=\mu_{2}$, and so the gradient-like points are those for which $\mu_{1} \neq \mu_{2}$ (which partially explains their name). Understanding the dynamical system then splits into two tasks: first, to understand the structure and dynamics within each $R_{\mu}$; and second, to understand the connections between the various $\boldsymbol{R}_{\mu}$. This second task is often accomplished by studying a partial order on $\left\{\boldsymbol{R}_{\mu}\right\}$ defined so that $R_{\mu_{1}}>R_{\mu_{2}}$ if there is a chain of orbits going from $R_{\mu_{1}}$ to $R_{\mu_{2}}$. 
In summary then, one distinguishes two types of orbits depending on their asymptotic behaviour. The point we are emphasizing here is that the asymptotic behaviour can be distinguished by the location of the $\alpha$ - and $\omega$-limit sets, and so, for example, $R_{\mu}=\left\{z: \alpha(z) \cup \omega(z) \subseteq R_{\mu}\right\}$. For maps of the annulus one has another tool for describing asymptotic behaviour, the forward and backward rotation sets. In analogy with the above, we distinguish two types of ' $\rho$-behaviour' based on the location of these two sets. If the connected components of $\rho(f)$ are $\left\{c_{\eta}\right\}$ for $\eta$ in some index set, then by the definition of $\rho(f)$ and the fact that $\rho(f)=\rho^{*}(f)$, one has that for each $z, \rho^{*}(z) \subseteq c_{\eta_{1}}$ and $\rho(z) \subseteq c_{\eta_{2}}$ for some $\eta_{1}$ and $\eta_{2}$. We think of $z$ as ' $\rho$-recurrent' if $\eta_{1}=\eta_{2}$ and ' $\rho$-gradient-like' otherwise. The analogue of a component of the chain recurrent set is then $\Lambda(c)=\left\{z: \rho(z) \cup \rho^{*}(z) \subseteq c\right\}$, where $c$ is a component of $\rho(f)$. We let $\Lambda_{f}$ be the union of the $\Lambda(c)$, i.e. $\Lambda_{f}$ is the ' $\rho$-recurrent' set. One studies the connections between the $\Lambda(c)$ by defining a partial order $\Lambda\left(c_{1}\right)>\Lambda\left(c_{2}\right)$ if there is a point $z$ with $\rho^{*}(z) \subseteq c_{1}$ and $\rho(z) \subseteq c_{2}$ and then extending the order to get transitivity. This is possible because of a no-cycle property that follows from lemma 3.2.

We shall show in theorem 3.3 that if $f$ is positive tilt, $\rho^{*}(z) \subseteq c$ if and only if $\alpha(z) \subseteq \Lambda(c)$, and $\rho(c) \subseteq c$ if and only if $\omega(z) \subseteq \Lambda(c)$, and so the parallels described above are more than an analogy. In particular, each chain recurrent point is $\rho$-recurrent (i.e. $R(f) \subseteq \Lambda_{f}$ ), and so $\rho$-gradient-like points are always gradient-like. In fact the decomposition into ' $\rho$-recurrent components' with the associated partial order is an example of a quasi-Morse decomposition (to be defined later).

From Conley's point of view the decomposition of a dynamical system into components of $R(f)$ (or $\Lambda_{f}$ ) is in general unsatisfactory because, among other things, $R(f)$ (or $\rho(f)$ ) may have infinitely many components and so the decomposition may not be stable under perturbations. Accordingly, one carefully collects together components of $R(f)$ and their connecting orbits to get a decomposition with a finite number of components, called a Morse decomposition, which will be stable. In our case we collect together the $\Lambda(c)$ and their ' $\rho$-connections' into sets of the form $\Lambda\left(c_{1}, c_{2}\right)=\left\{z: \rho(z) \cup \rho^{*}(z) \subseteq\left\langle c_{1} \cup c_{2}\right\rangle\right\}$. This is a good choice, since it turns out that if $\left\{c_{1}, c_{1}^{\prime}, \ldots, c_{k}, c_{k}^{\prime}\right\}$ is a collection of components of $\rho(f)$ with $\rho(f) \subseteq \bigcup_{i=1}^{k}\left\langle c_{i}, c_{i}^{\prime}\right\rangle$ as a disjoint union, then $\bigcup_{i=1}^{k} \Lambda\left(c_{i}, c_{i}^{\prime}\right)$ will be a Morse decomposition for $f$. From this point of view, then, understanding the twist map consists of studying the structure and dynamics within the $\Lambda\left(c_{i}, c_{i}^{\prime}\right)$ and understanding the connections between the $\Lambda\left(c_{i}, c_{i}^{\prime}\right)$.

Another notion which is useful in understanding Conley's decompositions is provided by attractor-repeller pairs. For the purpose of continuing our analogy between $\alpha(z)$ and $\rho^{*}(z)$, etc., we may think of an attractor-repeller pair $\left(X, X^{*}\right)$ as a splitting of the space into two disjoint compact invariant sets and their connecting orbits which may be characterized by

$$
X=\{z: \alpha(z) \cup \omega(z) \subseteq X\}, \quad X^{*}=\left\{z: \alpha(z) \cup \omega(z) \subseteq X^{*}\right\}
$$

and

$$
C\left(X, X^{*}\right)=M-\left\{X \cup X^{*}\right\}=\left\{z: \alpha(z) \subseteq X^{*} \text { and } \omega(z) \subseteq X\right\}
$$


For the $\rho$-analogue we split $\rho(f)$ into two pieces as a disjoint union,

$$
\rho(f) \subseteq\left[\rho\left(\partial_{1}\right), \sup c_{1}\right] \cup\left[\inf c_{2}, \rho\left(\partial_{2}\right)\right],
$$

and define a ' $\rho$-attractor-repeller pair' as

$$
Z^{*}=\left\{z: \rho(z) \cup \rho^{*}(z) \subseteq\left[\rho\left(\partial_{1}\right), \sup c_{1}\right]\right\}, \quad Z=\left\{Z: \rho(z) \cup \rho^{*}(z) \subseteq\left[\inf c_{2}, \rho\left(\partial_{2}\right)\right]\right\}
$$

and

$$
C\left(Z, Z^{*}\right)=\left\{z: \rho^{*}(z) \subseteq\left[\rho\left(\partial_{1}\right), \sup c_{1}\right] \text { and } \rho(z) \subseteq\left[\inf c_{2}, \rho\left(\partial_{2}\right)\right]\right\}
$$

We shall show in lemma 3.2 that such $Z$ and $Z^{*}$ actually are an attractor-repeller pair in the sense of Conley. In fact $Z^{*}=R^{\infty}\left(T^{-p} F^{q}\right)$ and $Z=L^{\infty}\left(T^{-p} F^{q}\right)$ for a $p / q$ with $\sup c_{2}<p / q<\inf c_{1}$ when $T^{-q} F^{q}$ is a down map. (Note that since $p / q \notin \rho(f)$, $T^{-p} F^{q}$ will be fixed point free.) Finally, in analogy with the fact that $R(f)=\cap$ $\left\{X \cup X^{*}:\left(X, X^{*}\right)\right.$ is an attractor-repeller pair $\}$, we have $\Lambda_{f}=\cup\left\{Z \cup Z^{*}:\left(Z, Z^{*}\right)\right.$ is a ' $\rho$-attractor-repeller pair'\}.

The first lemma will help in the description of the sets $\Lambda\left(c_{1}, c_{2}\right)$. If $\Lambda \subseteq A$ is a closed set, let $\tilde{\Lambda}_{+}=\left\{z \in \tilde{\Lambda}: I_{z}^{+} \cap \tilde{\Lambda}=\varnothing\right\}$. Thus $\tilde{\Lambda}_{+}$is the 'upper edge' of $\tilde{\Lambda}$. One defines $\tilde{\Lambda}_{-}$similarly. If $\partial_{2} \subseteq \Lambda^{c}$, a point $z \in \Lambda^{c}$ is said to be right accessible in $\Lambda^{c}$ if there is a positively tilted arc contained in $\Lambda^{c}$ that connects $z$ to $\partial_{2}$ (see figure 5). Lemma 3.1

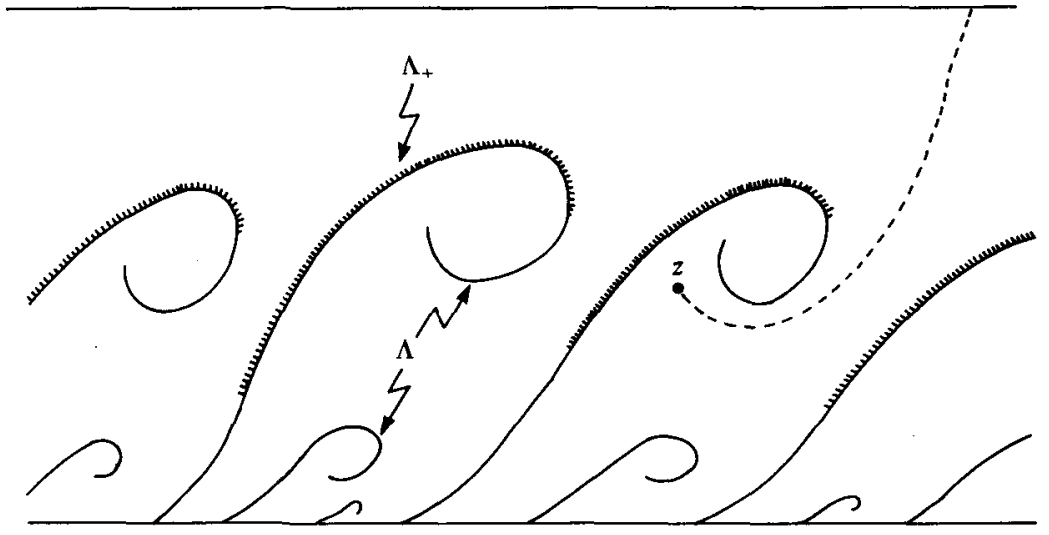

Figure 5

collects some results about invariant sets of positive tilt maps that are somewhat standard. Under suitable hypotheses on $\Lambda$, Birkhoff [8] shows that $\Lambda_{+}$is negatively invariant. Le Calvez [27] remarks that this implies the existence of an Aubry-Mather set $\Sigma$ inside $\Lambda_{+}$. The last statement of the lemma (similar to lemma 1 in [27]) roughly says that of all the points in $\Lambda$, those in $\Sigma$ move to the right most quickly under iteration.

LEMMA 3.1. If $f \in P T$ and $\Lambda$ is a compact connected invariant set with $\partial_{1} \subseteq \Lambda, \partial_{2} \subseteq \Lambda^{c}$, $\Lambda^{c}$ homeomorphic to a half-open annulus and each point of $\Lambda^{c}$ right accessible in $\Lambda^{c}$, then $f^{-1}\left(\mathrm{Cl}\left(\Lambda_{+}\right)\right) \subseteq \Lambda_{+}$and thus there exists an Aubry-Mather set $\Sigma \subseteq \Lambda_{+}$. In addition, 
if $z \in \Lambda$ and $p \in \Sigma$ with $\pi_{1}(z)<\pi_{1}(p)$, then $\pi_{1}(F(z))<\pi_{1}(F(p))$ and so, for all $z \in \Lambda$, $\rho(z) \subseteq\left[\rho\left(\partial_{1}\right), \rho(\Sigma)\right]$.

Proof. In [8, §9] Birkhoff shows under hypothesis similar to those in this lemma that for monotone twist maps $f, f^{-1}\left(\Lambda_{+}\right) \subseteq \Lambda_{+}$. His proof goes through with minor alterations for positive tilt maps $f$ to show that $f^{-1}\left(\mathrm{Cl}\left(\Lambda_{+}\right)\right) \subseteq \Lambda_{+}$. This implies that $\alpha\left(\Lambda_{+}\right) \subseteq \mathrm{Cl}\left(\Lambda_{+}\right)$and so $\alpha\left(\Lambda_{+}\right)=f^{-1}\left(\alpha\left(\Lambda_{+}\right)\right) \subseteq \Lambda_{+}$. Since $\alpha\left(\Lambda_{+}\right)$is compact and completely invariant, we may find a minimal set $\Sigma \subseteq \alpha\left(\Lambda_{+}\right)$. Now $\pi_{1}$ restricted to $\Sigma$ is injective since $\Sigma \subseteq \Lambda_{+}$. The other properties needed to show $\Sigma$ is link-monotone will be verified after we prove the last inequality in the theorem.

Assume this inequality is false. Then for some $p \in \Sigma$ and $z \in \Lambda, \pi_{1}(z)<\pi_{1}(p)$ but $\pi_{1}(F(z)) \geq \pi_{1}(F(p))$. We assume that $\pi_{1}(F(z))>\pi_{1}(F(p))$; the case with equality requires only minor alterations.

A main step in Birkhoff's proof referred to above is that under the hypothesis of this lemma, if $V=\left\{z \in \Lambda^{c}: I_{z}^{+} \subseteq \Lambda^{c}\right\}$, then $F^{-1}(V) \subseteq V$. Since $F(p) \subseteq \Sigma_{+}$, then $I_{F(p)}^{+} \subseteq$ $V$ and so $F^{-1}\left(I_{F(p)}^{+}\right) \subseteq V$. Now $F^{-1}\left(I_{F(p)}^{+}\right)$is a negatively tilted arc, so if $\pi_{2}\left(\right.$ bot $\left.\left(F^{-1}\left(I_{F(p)}\right) \cap I_{z}\right)\right)>\pi_{2}(z)$ or if $F^{-1}\left(I_{F(p)}\right) \cap I_{z}=\varnothing$, then using the analogue of lemma 2.1(a) for negatively tilted arcs, $z$ is topologically to the left of $F^{-1}\left(I_{F(p)}\right)$ and so $F(z)$ is topologically to the left of $I_{F(p)}$, a contradiction. On the other hand, if $\pi_{2}\left(\right.$ bot $\left.\left(F^{-1}\left(I_{F(p)}\right) \cap I_{z}\right)\right) \leq \pi_{2}(z)$, then since $\pi_{1}(z)<\pi_{1}(p), \pi_{2}\left(\right.$ bot $\left(F^{-1}\left(I_{F(p)}^{+}\right) \cap\right.$ $\left.\left.I_{z}\right)\right) \leq \pi_{2}(z)$ but $F^{-1}\left(I_{F(p)}^{+}\right) \subseteq V$, a contradiction, since $z \in \Lambda$. This proves the inequality.

By what we have just proven it is clear that $\Sigma$ has property (2) in the definition of link-monotone. The fact that $\Sigma$ has property (3) follows from property (2) and the fact that $f(\Sigma) \subseteq \Lambda_{+}$.

There are some variants of this theorem we wish to note for future use. First, if each point of $\Lambda^{c}$ is left accessible in $\Lambda^{c}$, then the same conclusions hold, except in this case $f\left(\mathrm{Cl}\left(\Lambda_{+}\right)\right) \subseteq \Lambda_{+}$. Second, if $\partial_{2} \subseteq \Lambda$ and $\partial_{1} \subseteq \Lambda^{c}$, call a point $z \in \Lambda^{c}$ left accessible if there is a positively tilted arc in $\Lambda^{c}$ connecting $z$ to $\partial_{1}$. In this case one gets an Aubry-Mather set $\Sigma \subseteq \Lambda_{-}$, and if $z \in \Lambda$ and $p \in \Sigma, \pi_{1}(z)>\pi_{1}(p)$ implies $\pi_{1}(F(z))>\pi_{1}(F(p))$ and so $\rho(z) \subseteq\left[\rho(\Sigma), \rho\left(\partial_{2}\right)\right]$. The same is true if we assume that each point in $\Lambda^{c}$ is right accessible in $\Lambda^{c}$.

We also note that if $F$ is a fixed point free positive tilt map, then $L^{\infty}(F)$ and $R^{\infty}(F)$ will satisfy the hypothesis of this theorem or one of the variants just mentioned. For example, if $F$ is a fixed point free down map and $U$ is as in the proof of theorem 2.3 , then we show there that $\left(R^{\infty}(F)\right)^{c}=\bigcup_{i=0}^{\infty} F^{-i}(U)$. It is clear from the definition of $U$ that $z \in U$ implies $I_{z}^{-} \subseteq U$, and since for all $i>0, F^{-i}\left(I_{z}\right)$ is a negatively tilted arc (since $F^{-1}$ is a 'negative tilt map'), one has that each point in $\left(R^{\infty}\right)^{c}$ is right accessible in $\left(R^{\infty}\right)^{c}$. The other necessary hypotheses also follow from theorem 2.3 .

For the next lemma we need to take another idea from Conley [17, II $\S 5 . B]$. We again leave it to the reader to make the appropriate alterations of his proof to make it suitable for the homeomorphism case. If $M$ is a compact metric space and $f: M \rightarrow M$ a homeomorphism with an attractor-repeller pair $\left(X, X^{*}\right)$, then there 
always exists a continuous $\psi: M \rightarrow[0,1]$ (called a Liapounov function) with $\psi^{-1}(0)=$ $X, \psi^{-1}(1)=X^{*}$ and for $x \in C\left(X, X^{*}\right), \psi(f(x))<\psi(x)$.

We also need a fact about attractor-repeller pairs that is slightly different for homeomorphisms than for flows. If $f: M \rightarrow M$ is a homeomorphism and $\left(X, X^{*}\right)$ is an attractor-repeller pair for $f^{q}$ for some $q>0$, then $\left(X \cap f(X) \cap \cdots \cap f^{q-1}(X)\right.$, $\left.X^{*} \cup f\left(X^{*}\right) \cup \cdots \cup f^{q-1}\left(X^{*}\right)\right)$ are an attractor-repeller pair for $f$ (note that $\left(X, X^{*}\right)$ may not be).

Since $\rho(f)$ is a closed subset of twist $(f)$, its complement in twist $(f)$ is a countable union of open intervals called complementary gaps of $\rho(f)$ and denoted $\left\{J_{i}\right\}$. Now if $p / q$ is contained in some $J_{i}$, then by theorem $1.2(\mathrm{a}), T^{-p} F^{q}$ is fixed point free so by theorem 2.3 it is either up or down. Part of the content of the next lemma is that if we choose another rational, say $r / s$, contained in $J_{i}$, then $T^{-r} F^{s}$ will have the same orientation as $T^{-p} F^{q}$. This allows us to unambiguously label each complementary gap of $\rho(f)$ as either an up gap or a down gap.

For each rational $p / q$ in twist $(f)$, define $R^{\infty}(p / q)=R^{\infty}\left(T^{-p} F^{q}\right)$ and $L^{\infty}(p / q)=$ $L^{\infty}\left(T^{-p} F^{q}\right)$. Finally, if $\Gamma_{1}$ and $\Gamma_{2}$ are disjoint, homotopically non-trivial circles in the annulus, let $A\left(\Gamma_{1}, \Gamma_{2}\right)$ denote the annulus bounded by these two circles.

LEMMA 3.2. Let $f: A \rightarrow A$ be a positive tilt map and $J$ be a complementary gap of $\rho(f)$. Fix $a p / q \in J$ and let $R^{\infty}(J)=R^{\infty}(p / q)$ and $L^{\infty}(J)=L^{\infty}(p / q)$ and assume that $T^{-p} F^{q}$ is a down map, then for all $r / s \in J, T^{-s} F^{r}$ is a down map and $R^{\infty}(r / s)=R^{\infty}(J)$ and $L^{\infty}(r / s)=L^{\infty}(J)$. In addition, $L^{\infty}(J)$ and $R^{\infty}(J)$ are an attractor-repeller pair for $f$ and

and

$$
\begin{aligned}
L^{\infty}(J) & =\left\{z: \rho(z) \cup \rho^{*}(z) \subseteq\left[\rho\left(\partial_{1}\right), \text { inf } J\right]\right\} \\
& =\left\{z: \pi_{1}\left(F^{s}(x)\right)-\pi_{1}(x)<r \text { for all } x \in o(z) \text { and } r / s>\inf J\right\}
\end{aligned}
$$

$$
\begin{aligned}
R^{\infty}(J) & =\left\{z: \rho(z) \cup \rho^{*}(z) \subseteq\left[\sup J, \rho\left(\partial_{2}\right)\right]\right\} \\
& =\left\{z: \pi_{1}\left(F^{s}(x)\right)-\pi_{1}(x)>r \text { for all } x \in o(z) \text { and } r / s<\sup J\right\} .
\end{aligned}
$$

Further, for $z \in A, \alpha(z) \subseteq R^{\infty}(J)$ if and only if $\rho^{*}(z) \subseteq\left[\sup J, \rho\left(\partial_{2}\right)\right]$, and $\omega(z) \subseteq L^{\infty}(J)$ if and only if $\rho(z) \subseteq\left[\rho\left(\partial_{1}\right)\right.$, inf $\left.J\right]$, and so $C\left(R^{\infty}(J), L^{\infty}(J)\right)=\left\{z: \rho(z) \subseteq\left[\rho\left(\partial_{1}\right)\right.\right.$, inf $\left.J\right]$ and $\left.\rho^{*}(z) \subseteq\left[\sup J, \rho\left(\partial_{2}\right)\right]\right\}$. Finally, $C\left(R^{\infty}(J), L^{\infty}(J)\right)$ is homeomorphic to an open annulus that is homotopically non-trivial in $A$ and there exists a homotopically non-trivial embedded circle $\Gamma(J) \subseteq C\left(R^{\infty}, L^{\infty}\right)$ such that $f(\Gamma(J)) \subseteq \operatorname{Int}\left(A\left(\Gamma(J), \partial_{1}\right)\right)$, and so $L^{\infty}$ is the largest invariant set in $A\left(\partial_{1}, \Gamma(J)\right)$ and $R^{\infty}$ is the largest invariant set in $\boldsymbol{A}\left(\Gamma(J), \partial_{2}\right)$.

Proof. We begin with a few facts that will be used in the sequel, often without comment. If $x \in L^{\infty}(p / q)$, then it is clear that $\rho\left(x, T^{-p} F^{q}\right) \cup \rho^{*}\left(x, T^{-p} F^{q}\right) \subseteq(-\infty, 0)$, and if $x \in R^{\infty}(p / q)$, then $\rho\left(x, T^{-p} F^{q}\right) \cup \rho^{*}\left(x, T^{-p} F^{q}\right) \subseteq(0, \infty)$. Since we are assuming that $T^{-p} F^{q}$ is a fixed point free down map, theorem 2.3 yields that $\rho^{*}\left(x, T^{-p} F^{q}\right) \subseteq$ $(-\infty, 0)$ implies that $x \in L^{\infty}(p / q)$. Thus, using lemma 1.1, we have

Similarly,

$$
L^{\infty}(p / q)=\left\{z: \rho(z, f) \cup \rho^{*}(z, f) \subseteq\left[\rho\left(\partial_{1}\right), p / q\right)\right\} .
$$

$$
R^{\infty}(p / q)=\left\{z: \rho(z, f) \cup \rho^{*}(z, f) \subseteq\left(p / q, \rho\left(\partial_{2}\right)\right]\right\}
$$

and

$$
C\left(R^{\infty}(p / q), L^{\infty}(p / q)\right)=\left\{z: \rho(x, f) \subseteq\left(p / q, \rho\left(\partial_{2}\right)\right] \text { and } \rho^{*}(x, f) \subseteq\left[\rho\left(\partial_{1}\right), p / q\right)\right\}
$$


Theorem 2.3 implies that $L^{\infty}(p / q)$ and $R^{\infty}(p / q)$ are an attractor-repeller pair for $f^{q}$. We now show they are also an attractor-repeller pair for $f$. The first step is to prove that they are both $f$-invariant. Say there existed an $x \in$ $R^{\infty}(p / q)-f\left(R^{\infty}(p / q)\right)$, then $\rho(x) \subseteq\left[\rho\left(\partial_{1}\right), p / q\right)$, and since $f^{-1}(x) \notin R^{\infty}(p / q)$, $\rho\left(f^{-1}(x)\right) \subseteq\left(p / q, \rho\left(\partial_{2}\right)\right]$, a contradiction, since $\rho(x)=\rho\left(f^{-1}(x)\right)$. One obtains a similar contradiction if $f\left(R^{\infty}(p / q)\right)-R^{\infty}(p / q) \neq \varnothing$, and a similar proof shows that $f\left(L^{\infty}\right)=L^{\infty}$. Thus, by our comment above the lemma, $R^{\infty}(p / q)=R^{\infty}(p / q) \cup \cdots \cup$ $f^{q-1}\left(R^{\infty}(p / q)\right)$ and $L^{\infty}(p / q)=L^{\infty}(p / q) \cap \cdots \cap f^{q-1}\left(L^{\infty}(p / q)\right)$ are an attractorrepeller pair for $f$.

Next we show that for $r / s \in J, R^{\infty}(r / s)=R^{\infty}(p / q)$. Say there existed an $x \in R^{\infty}(r / s)-R^{\infty}(p / q)$, then $\rho(x) \subseteq\left(r / s, \rho\left(\partial_{2}\right)\right]$ and $\rho(x) \subseteq\left[\rho\left(\partial_{1}\right), p / q\right)$. Now $p / q<r / s$ is clearly impossible, and if $r / s<p / q$, then $\rho(x) \subseteq(r / s, p / q)$ but $(r / s, p / q) \cap \rho(f)=\varnothing$, a contradiction. Now assume there exists an $x \in$ $R^{\infty}(p / q)-R^{\infty}(r / s)$. Since $T^{-r} F^{s}$ is also a fixed point free map, the argument of the previous paragraph shows that $R^{\infty}(r / s)$ is an attractor or repeller for $f$. If it is an attractor, one uses the argument just given. If it is a repeller, then $\rho^{*}(x) \subseteq$ $\left(p / q, \rho\left(\partial_{2}\right)\right]$ and $\rho^{*}(x) \subseteq\left[\rho\left(\partial_{1}\right), r / s\right)$ and proceed as above. Thus $R^{\infty}(r / s)=$ $R^{\infty}(p / q)$ and they are both repellers, and thus their complementary attractors are equal and so $L^{\infty}(r / s)=L^{\infty}(p / q)$. From this and the facts of the first paragraph of the proof one gets the characteristics of $R^{\infty}(J), L^{\infty}(J)$ and $C\left(R^{\infty}(J), L^{\infty}(J)\right)$ in terms of the behaviour of $\rho(x, f)$ and $\rho^{*}(x, f)$. Using these characteristics, $\rho^{*}(z) \subseteq$ [sup $\left.J, \rho\left(\partial_{2}\right)\right]$ if and only if $z \in R^{\infty}(J) \cup C\left(R^{\infty}(J), L^{\infty}(J)\right)$, which happens if and only if $\alpha(z) \subseteq R^{\infty}(J)$ since $L^{\infty}(J)$ and $R^{\infty}(J)$ are an attractor-repeller pair.

As noted after the proof of lemma 3.1, $L^{\infty}(J)$ satisfies the hypothesis of that lemma and so there is an Aubry-Mather set $\Sigma_{+}$contained in the upper edge of $L^{\infty}(J)$, and for $x \in L^{\infty}(J)$ and $p \in \Sigma_{+}, \pi_{1}(x)<\pi_{1}(p)$ implies that $\pi_{1}(F(x))<\pi_{1}(F(p))$. Now say there existed an $x \in L^{\infty}(J)$ and some $r / s>\inf J$ with $\pi_{1}\left(F^{s}(x)\right)-\pi_{1}(x) \geq r$. Pick a $p \in \Sigma_{+}$with $\pi_{1}(x)<\pi_{1}(p)$, and by repeated application of the inequality above, $\pi_{1}\left(F^{s}(p)\right)-\pi_{1}(p) \geq r$. Now if all $p \in \Sigma_{+}$satisfy this inequality, then by induction, $\rho\left(\Sigma_{+}\right) \geq r / s$. On the other hand, if for some $p^{\prime} \in$ $\Sigma_{+}, \pi_{1}\left(F^{s}\left(p^{\prime}\right)\right)-\pi_{1}\left(p^{\prime}\right)<r$, then since $\Sigma_{+}$is Aubry-Mather, $\rho\left(\Sigma_{+}\right)=r / s$. But since $\Sigma_{+} \subseteq L^{\infty}(J), \rho\left(\Sigma_{+}\right) \subseteq\left[\right.$ inf $\left.J, \rho\left(\partial_{2}\right)\right]$, contradicting the assumption $r / s>\inf J$. We have therefore proven $L^{\infty}(J) \subseteq\left\{z: \pi_{1}\left(F^{s}(x)\right)-\pi_{1}(x)<r\right.$ for all $x \in o(z)$ and $\left.r / s>\inf J\right\}$. The other inclusion is easy and the proof for $R^{\infty}(J)$ is similar.

Since $L^{\infty}(J)$ and $R^{\infty}(J)$ are an attractor-repeller pair for $f$, as noted above the theorem, we may find a Liapounov function for the pair, $\psi^{\prime}: A \rightarrow[0,1]$. By making a small $C^{0}$-perturbation of $\psi^{\prime}$, supported on $\psi^{-1}\left(\frac{1}{5}, \frac{4}{5}\right)$, we may obtain another Liapounov function for the pair $\psi$, with $\psi$ smooth on $\psi^{-1}\left(\frac{2}{5}, \frac{3}{5}\right)$. Using Sard's theorem, find a regular value $p$ of $\psi$ with $p \in\left(\frac{2}{5}, \frac{3}{5}\right)$. Since $\partial_{1} \subseteq R^{\infty}(J)$ and $\partial_{2} \subseteq L^{\infty}(J), \phi^{-1}(p)$ must contain a homotopically non-trivial embedded circle $\Gamma \subseteq C\left(R^{\infty}(J), L^{\infty}(J)\right)$. Since $\psi$ decreases in orbits there, $f(\Gamma) \cap \Gamma=\varnothing$, and since $L^{\infty}$ is an attractor, $f(\Gamma) \subseteq$ Int $\left(A\left(\Gamma, \partial_{1}\right)\right)$. The last two statements of the theorem are easy consequences of the above. 
Similar techniques to those in this proof show the following. If $f: A \rightarrow A$ is a homeomorphism that has the circle intersection property (i.e. for each homotopically non-trivial circle $\Gamma, f(\Gamma) \cap \Gamma \neq \varnothing)$, then so does $f^{q}$ for all $q>0$. Here is a sketch of the proof. If $f^{q}$ does not have the circle intersection property for some $q$, let $\Gamma$ be a circle with $f^{q}(\Gamma) \cap \Gamma=\varnothing$. Then $X_{j}=\bigcap_{i=1}^{\infty} f^{i q}\left(A\left(\Gamma, \partial_{j}\right)\right)$ will be an attractor for $f^{q}$ when $j$ is chosen so that $f(\Gamma)$ is inside $A\left(\Gamma, \partial_{j}\right)$. But then $X_{1} \cup f\left(X_{1}\right) \cup \cdots \cup f^{q-1}\left(X_{1}\right)$ and $X_{2} \cap f\left(X_{2}\right) \cap \cdots \cap f^{q-1}\left(X_{2}\right)$ will be an attractor-repeller (or repeller-attractor) pair for $f$, and we use the Liapounov function of this pair as in the proof above to get a $\Gamma^{\prime}$ with $f\left(\Gamma^{\prime}\right) \cap \Gamma^{\prime}=\varnothing$.

Before stating our main theorem, we need to give a precise definition of the notion of a Morse decomposition discussed in the introduction to this section. Let $M$ be a compact metric space and $g: M \rightarrow M$ be a homeomorphism. A quasi-Morse decomposition for $g$ is a collection of compact $g$-invariant sets, $N_{\mu}$, for $\mu$ in some index set, which are equipped with a partial order that has the property that for all $x \in M$ either (1) $x \in N_{\mu}$ for some $\mu$ or (2) there exists $N_{\mu_{1}}>N_{\mu_{2}}$ with $\alpha(x) \subseteq N_{\mu_{1}}$ and $\omega(x) \subseteq N_{\mu_{2}}$. If the index set is finite, then $\left\{N_{\mu}\right\}$ is called a Morse decomposition. As noted above, this case is preferable, because as a consequence of the duality between Morse decompositions and attractor filtrations, a Morse decomposition will continue to nearby maps.

We shall also need some notions associated with the decomposition of $\rho(f)$ into components and complementary gaps. Fix an $f \in P T$. A collection of pairs of components of $\rho(f),\left\{\left(c_{\eta}, c_{\eta}^{\prime}\right): \eta \in B\right\}$, will be called a covering family of pairs if for each $\eta, \sup c_{\eta} \leq \sup c_{\eta}^{\prime}$ and $\bigcup_{\eta \in B}\left\langle c_{\eta} \cup c_{\eta}^{\prime}\right\rangle$ is a disjoint union that contains $\rho(f)$. We define a partial order (called the gap order) on the family as follows. By construction, $\cup\left\langle c_{\eta} \cup c_{\eta}^{\prime}\right\rangle$ is compact so its complement is the union of open intervals called the complementary gaps of the family; we say that $\left(c_{\eta_{1}}, c_{\eta_{1}}^{\prime}\right)>\left(c_{\eta_{2}}, c_{\eta_{2}}^{\prime}\right)$ if for any gap $J$ complementary to the family, $\sup c_{\eta_{1}}^{\prime} \leq \inf J \leq \inf c_{\eta_{2}}$ implies that $J$ is an up gap, and that $\left(c_{\eta_{1}}, c_{\eta_{1}}^{\prime}\right)<\left(c_{\eta_{2}}, c_{\eta_{2}}^{\prime}\right)$ if any such gap is a down gap. More informally, one pair is bigger than another means that all the gaps between them are oriented from the larger to the smaller. In the obvious way, this partial order induces one on $\left\{\Lambda\left(c_{\mu}, c_{\eta}^{\prime}\right): \eta \in B\right\}$ also called the gap order. (Recall that $\Lambda\left(c_{1}, c_{2}\right)=$ $\left\{z: \rho(z) \cup \rho^{*}(z) \subseteq\left\langle c_{1} \cup c_{2}\right\rangle\right\}$ and $\Lambda(c)=\Lambda(c, c)$.)

Part (a) of the next theorem says that this family with the gap order is a quasi-Morse decomposition. The point here is that the data giving the components of $\rho(f)$ and the orientation of the complementary gaps suffice to determine a Morse decomposition. Part (b) of the theorem shows how this decomposition is situated in the annulus. It could be interpreted as giving a means of constructing a filtration of the annulus by sub-annuli. Part (c) gives more information about the structure of the (quasi) Morse sets $\Lambda\left(c_{1}, c_{2}\right)$, and part (d) gives a few applications of the correlation of the location of $\alpha$ - and $\omega$-limit sets to the value of $\rho^{*}$ and $\rho$.

THEOREM 3.3. Let $f \in P T$ with $\left\{c_{\mu}\right\}$ and $\left\{J_{i}\right\}$ the connected components and complementary gaps of $\rho(f)$ respectively. 
(a) If $\left\{\left(c_{\eta}, c_{\eta}^{\prime}\right): \eta \in B\right\}$ is a covering family of component pairs, then

$$
\left\{\Lambda\left(c_{\eta}, c_{\eta}^{\prime}\right): \eta \in B\right\}
$$

with the gap order is a quasi-Morse decomposition for $f$. In particular, if the index set $B$ is finite, it is a Morse decomposition and $\{\Lambda(c): c$ is a component of $\rho(f)\}$ with the gap order is a quasi-Morse decomposition.

(b) For each complementary gap $J_{i}$ there exists a homotopically non-trivial embedded circle $\Gamma\left(J_{i}\right)$ with the property that if $\sup J_{1}<\inf J_{2}$, then $\Gamma\left(J_{1}\right) \subseteq \operatorname{Int}\left(A\left(\Gamma\left(J_{2}\right), \partial_{1}\right)\right)$ and $\left\langle c_{1} \cup c_{2}\right\rangle \subseteq\left\langle J_{1} \cup J_{2}\right\rangle$ if and only if $\Lambda\left(c_{1}, c_{2}\right) \subseteq \operatorname{Int}\left(A\left(\Gamma\left(J_{1}\right), \Gamma\left(J_{2}\right)\right)\right)$. Also, if $J$ is a down gap, $f(\Gamma(J)) \subseteq \operatorname{Int}\left(A\left(\Gamma(J), \partial_{1}\right)\right)$, and if $J$ is an up gap, $f(\Gamma(J)) \subseteq \operatorname{Int}\left(A\left(\Gamma(J), \partial_{2}\right)\right)$.

(c) Given two components of $\rho(f), c_{1}$ and $c_{2}$, with $\sup c_{1} \leq \sup c_{2}$, then $\Lambda\left(c_{1}, c_{2}\right)=$ $\left\{z: \forall x \in o(z), \pi_{1}\left(F^{s}(x)-x\right)<r\right.$ for all $r / s>\sup c_{2}$ and $\pi_{1}\left(F^{s}(x)-x\right)>r$ for all $\left.r / s<\inf c_{1}\right\}$. In addition, the upper and lower edges of $\Lambda\left(c_{1}, c_{2}\right)$ contain Aubry-Mather sets $\Sigma_{+}$and $\Sigma_{-}$respectively with $\rho\left(\Sigma_{+}\right)=\sup c_{2}$ and $\rho\left(\Sigma_{-}\right)=\inf c_{1}$. Further, if $p_{-} \in \Sigma_{-}$ and $p_{+} \in \Sigma_{+}$and $z \in \Lambda\left(c_{1}, c_{2}\right)$ and $\pi_{1}\left(p_{-}\right)<\pi_{1}(z)<\pi_{1}\left(p_{+}\right)$, then $\pi_{1}\left(F\left(p_{-}\right)\right)<$ $\pi_{1}(F(z))<\pi_{1}\left(F\left(p_{+}\right)\right)$.

(d) If $\Lambda_{f}=\cup\{\Lambda(c): c$ is a component of $\rho(f)\}$, then $\Lambda_{f}$ is compact invariant and contains the chain recurrent set of $f$. If $c$ is any component of $\rho(f)$, then $\rho(z) \subseteq c$ if and only if $\omega(z) \subseteq \Lambda(c)$, and $\rho^{*}(z) \subseteq c$ if and only if $\alpha(z) \subseteq \Lambda(c)$. If $z$ is a chain recurrent point with $\rho(z) \subseteq c$, then $z \in \Lambda(c)$ and $\rho^{*}(z) \subseteq c$. Finally, if $Z$ is a chain transitive set with $\left(\rho^{*}(Z) \cup \rho(Z)\right) \cap c \neq \varnothing$, then $Z \subseteq \Lambda(c)$ and $\rho^{*}(Z) \cup \rho(Z) \subseteq c$.

Proof. We prove the result of (c) first. Letting

$$
\Lambda\left(\partial_{1}, c_{2}\right)=\left\{z: \rho(z) \cup \rho^{*}(z) \subseteq\left\langle\rho\left(\partial_{1}\right) \cup c_{2}\right\rangle\right\}
$$

and defining $\Lambda\left(c_{2}, \partial_{2}\right)$ similarly and using lemma 3.2, we have

$$
\begin{aligned}
\Lambda\left(\partial_{1}, c_{2}\right) & =\cap\left\{L^{\infty}(J): \inf J \geq \sup c_{2}\right\} \\
& =\left\{z: \pi_{1}\left(F^{s}(x)\right)-\pi_{1}(x)<r \text { for all } x \in o(z) \text { and } r / s>\sup c_{2}\right\}
\end{aligned}
$$

and

$$
\begin{aligned}
\Lambda\left(c_{1}, \partial_{2}\right) & =\cap\left\{R^{\infty}(J): \sup J \leq \inf c_{1}\right\} \\
& =\left\{z: \pi_{1}\left(F^{s}(x)\right)-\pi_{1}(x)>r \text { for all } x \in o(z) \text { and } r / s<\inf c_{1}\right\} .
\end{aligned}
$$

Since $\Lambda\left(c_{1}, c_{2}\right)=\Lambda\left(c_{1}, \partial_{2}\right) \cap \Lambda\left(\partial_{1}, c_{2}\right)$, the result of the first sentence of (c) follows and, in addition, each $\Lambda\left(c_{1}, c_{2}\right)$ is compact.

As noted after lemma 3.1, for each $J, L^{\infty}(J)$ satisfies the hypothesis of that lemma. Now if $\sup J_{1}<\inf J_{2}$, then $L^{\infty}\left(J_{1}\right) \subseteq L^{\infty}\left(J_{2}\right)$ and this implies that $\Lambda\left(\partial_{1}, c_{2}\right)$ also satisfies the hypothesis of lemma 3.1, and so there exists an Aubry-Mather set $\Sigma_{+} \subseteq \Lambda_{+}\left(\partial_{1}, c_{2}\right)$. Using theorem 2.2, there is a (perhaps different) Aubry-Mather set $\Sigma^{\prime}$ with $\rho\left(\Sigma^{\prime}\right)=\rho^{*}\left(\Sigma^{\prime}\right)=$ sup $c_{2}$. This implies that $\Sigma^{\prime} \subseteq \Lambda\left(\partial_{1}, c_{2}\right)$, and so using lemma 3.1 again, $\rho\left(\Sigma^{\prime}\right) \subseteq\left[\rho\left(\partial_{1}\right), \rho\left(\Sigma_{+}\right)\right]$and so $\rho\left(\Sigma_{+}\right)=\rho\left(\Sigma^{\prime}\right)=\sup c_{2}$.

We now show that $\Sigma_{+} \subseteq \Lambda_{+}\left(c_{1}, c_{2}\right)$. Since $\Sigma_{+}$is Aubry-Mather, $\rho\left(\Sigma_{+}\right)=\rho^{*}\left(\Sigma_{+}\right)=$ sup $c_{2}$ and so $\Sigma_{+} \subseteq \Lambda\left(c_{1}, c_{2}\right)$. Since $\Lambda\left(c_{1}, c_{2}\right) \subseteq \Lambda\left(\partial_{1}, c_{2}\right)$, It is obvious that $I_{p}^{+} \cap$ $\Lambda\left(\partial_{1}, c_{2}\right)=\varnothing$ implies $I_{p}^{+} \cap \Lambda\left(c_{1}, c_{2}\right)=\varnothing$ and so $\Lambda_{+}\left(\partial_{1}, c_{2}\right) \cap \Lambda\left(c_{1}, c_{2}\right) \subseteq \Lambda_{+}\left(c_{1}, c_{2}\right)$, which implies that $\Sigma_{+} \subseteq \Lambda_{+}\left(c_{1}, c_{2}\right)$. A similar argument yields an Aubry-Mather $\Sigma_{-} \subseteq \Lambda_{-}\left(c_{1}, c_{2}\right)$ with $\rho\left(\Sigma_{-}\right)=\inf c_{1}$. The last sentence of (c) now follows from lemma 
3.1. It is now clear that $\Lambda_{f}=\cap\left\{L^{\infty}(J) \cup R^{\infty}(J): J\right.$ is a complementary gap of $\left.\rho(f)\right\}$, and since $L^{\infty}(J)$ and $R^{\infty}(J)$ are always an attractor-repeller pair, we have that $\Lambda_{f}$ is compact and contains the chain recurrent set of $f$ as needed for (d). In addition, it implies that if $Z$ is chain transitive and $Z \cap \Lambda(c) \neq \varnothing$ for some component $c$, then $Z \subseteq \Lambda(c)$, which implies the last statement in (d). The proof of the statement for a chain recurrent point $z$ is similar. Finally, since $\omega(z)$ is chain transitive for any point $z$, by what we have just proved, $\omega(z) \subseteq \Lambda(c)$ for some component $c$, and so using lemma 1.1(b), $\rho(z) \subseteq\langle\rho(\omega(z))\rangle \subseteq c$, which implies that $\rho(z) \subseteq c$ if and only if $\omega(z) \subseteq \Lambda(c)$. The statement involving $\rho^{*}(z)$ and $\alpha(z)$ has an identical proof.

To prove (a), we have already seen that each $\Lambda\left(c_{\eta}, c_{\eta}^{\prime}\right)$ is compact so we need to check that the gap order satisfies the appropriate properties. For this, choose a $z$ in the complement of $\cup \Lambda\left(c_{\eta}, c_{\eta}^{\prime}\right)$, then there are $\eta_{1} \neq \eta_{2}$ with $\rho(z) \subseteq\left\langle c_{\eta_{1}} \cup c_{\eta_{1}}^{\prime}\right\rangle$ and $\rho^{*}(z) \subseteq\left\langle c_{\eta_{2}} \cup c_{\eta_{2}}^{\prime}\right\rangle$. Using lemma 3.2, this implies that every gap between these two convex hulls must be oriented from $\left\langle c_{\eta_{2}} \cup c_{\eta_{2}}^{\prime}\right\rangle$ towards $\left\langle c_{\eta_{1}} \cup c_{\eta_{1}}^{\prime}\right\rangle$, i.e. $\left.\Lambda\left(c_{\eta_{2}}, c_{\eta_{2}}^{\prime}\right)\right\rangle$ $\Lambda\left(c_{\eta_{1}}, c_{\eta_{1}}^{\prime}\right)$ in the gap order. The previous sentence also implies, using (d), that $\omega(z) \subseteq \Lambda\left(c_{\eta_{2}}, c_{\eta_{2}}^{\prime}\right)$ and $\alpha(z) \subseteq \Lambda\left(c_{\eta_{1}}, c_{\eta_{1}}^{\prime}\right)$, finishing the proof of (a).

To prove the results of (b), we take another idea from Conley [16, II§ 6.4] and construct a Liapounov function, $\Psi: A \rightarrow[0,1]$, that distinguishes the various $\Lambda(c)$. For each complementary gap $J_{i}$, let $\psi_{i}$ be the Liapounov function for the pair, $R^{\infty}\left(J_{i}\right)$ and $L^{\infty}\left(J_{i}\right)$, constructed in the proof of lemma 3.2 and let $\Psi(x)=$ $\sum_{i=1}^{\infty} 2 \times 3^{-i} \psi_{i}(x)$. By the properties of the $\psi_{i}$ and the characterization of $\Lambda(c)$ as the intersections of various $L^{\infty}\left(J_{i}\right)$ and $R^{\infty}\left(J_{i}\right)$ given in the first paragraph of the proof, $\Psi$ is continuous, $x \in \Lambda_{f}$ implies $\Psi(x)=\Psi(f(x)), x \notin \Lambda_{f}$ implies $\Psi(f(x))<\Psi(x)$ and $\Psi$ separates the various $\Lambda(c)$ in the sense that if $x \in \Lambda\left(c_{1}\right)$ and $x_{2} \in \Lambda\left(c_{2}\right)$, then $\Psi\left(x_{1}\right)=\Psi\left(x_{2}\right)$ if and only if $c_{1}=c_{2}$. Moreover, $\Psi\left(\Lambda_{f}\right)$ is contained in the usual Cantor middle third set and is compact since $\Lambda_{f}$ is compact.

Now let $\left\{I_{i}\right\}$ be the complementary open gaps of $\Psi\left(\Lambda_{f}\right)$ and for each $i$ choose an open interval $I_{i}^{\prime}$ with $\mathrm{Cl}\left(I_{i}^{\prime}\right) \subseteq I_{i}$. By making a small $C^{0}$-perturbation (if necessary) in a small neighbourhood of $\Psi^{-1}\left(\cup I_{i}^{\prime}\right)$, we may assume that $\Psi$ is smooth on $\Psi^{-1}\left(\cup I_{i}^{\prime}\right)$. Given a complementary gap $J$ of $\rho(f)$, let $c_{1}$ and $c_{2}$ be such that sup $c_{1}=\inf J$ and $\sup J=\inf c_{2}$ and find a $I_{k}^{\prime} \subseteq\left\langle\Psi\left(\Lambda\left(c_{1}\right)\right), \Psi\left(\Lambda\left(c_{2}\right)\right)\right\rangle$. Now pick a regular value of $\Psi, p_{k} \in I_{k}^{\prime}$, and arguing as in the proof of lemma 3.2, we may find a homotopically non-trivial embedded circle $\Gamma(J) \subseteq \Psi^{-1}\left(p_{k}\right) \cap C\left(R^{\infty}(J), L^{\infty}(J)\right)$. The various facts given in (b) are a straightforward consequence of this construction of $\Gamma(J)$, the properties of $\Lambda\left(c_{1}, c_{2}\right)$ given in (c) (and its proof) and lemma 3.2 .

Our first remark is that the gap order on $\{\Lambda(c)\}$ may not be the finest order that makes the collection a quasi-Morse decomposition, i.e. it is not always the natural order. Thus if $\Lambda\left(c_{1}\right)>\Lambda\left(c_{2}\right)$, there may not be an orbit with $\alpha(z) \subseteq \Lambda\left(c_{1}\right)$ and $\omega(z) \subseteq \Lambda\left(c_{2}\right)$ even if $c_{1}$ and $c_{2}$ are adjacent components (by this we mean there is a gap $J$ with inf $c_{1}=\sup J$ and $\sup c_{2}=\inf J$ ). We will give an example of this shortly. If all the $\Lambda(c)$ separate, then the gap order will be the natural one.

We next give an example of an $f$ for which $\sup J_{1}=p / q=\inf J_{2}$, where $J_{1}$ and $J_{2}$ are complementary gaps of $\rho(f)$. This example is essentially that of the remarkable 


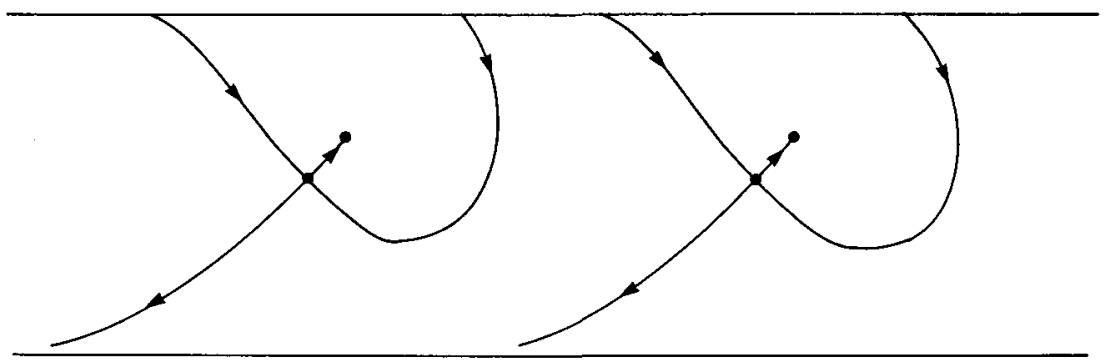

(a)

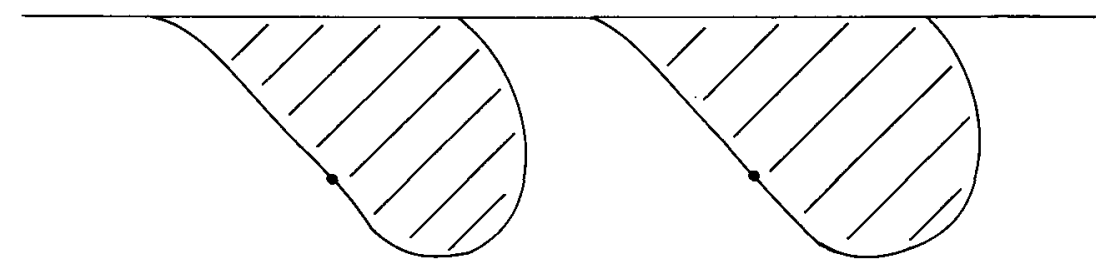

(b)

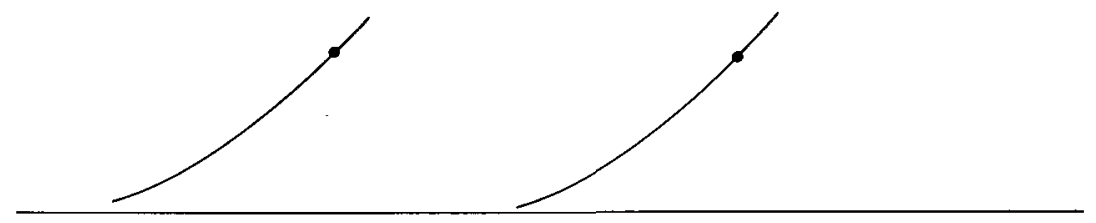

(c)

Figure 6

region $g$ in [2] (see also [25] and [13]). In figure 6(a) we show a sink-saddle pair with rotation number $p / q$, along with their stable and unstable manifolds. By sketching vertical lines and their images, one can verify that a fixed point with this configuration can exist for a monotone twist map. To create a $p / q$-periodic orbit with the desired behaviour, translate this construction by all integer multiples of $p / q$ and let the map permute the sinks and saddles in the appropriate manner. This configuration implies that $J_{1}$ and $J_{2}$ are down gaps, and in figure $6(\mathrm{~b})$ we show $R^{\infty}\left(J_{1}\right)$ and in figure $6(\mathrm{c})$ we show $L^{\infty}\left(J_{2}\right)$. Since $\Lambda(p / q)=R^{\infty}\left(J_{1}\right) \cap L^{\infty}\left(J_{2}\right)$, it is therefore equal to the sink, saddle and the portion of the unstable manifold of the saddle connecting them. By putting two examples of this type on top of each other and arranging it so the unstable manifolds of the upper saddle do not intersect the stable manifolds of the lower, one obtains the example referred to in the last paragraph.

One may also construct a non-separating $\Lambda(c)$ for the case $c$ is an interval by altering Birkhoff's construction of the attractor that bears his name [8] as follows. 
Let $g: A \rightarrow A$ be an area-preserving monotone twist map with no homotopically non-trivial invariant circles and let $o(\beta)$ be a non-monotone periodic orbit for $g$ (such orbits always exist by [12]). Birkhoff showed that maps such as $g$ have the boundary transit property, i.e. given neighbourhoods $U_{1}$ and $U_{2}$ of $\partial_{1}$ and $\partial_{2}$ respectively, there exists $z_{1} \in U_{1}$ and $z_{2} \in U_{2}$ and $n_{1}, n_{2} \in N$ with $f^{n_{1}}\left(z_{1}\right) \subseteq U_{2}$ and $f^{n_{2}}\left(z_{2}\right) \subseteq$ $U_{1}$. Now choose smooth functions $\alpha_{1}, \alpha_{2}: \mathbf{R} \rightarrow[0,1]$ so that graph $\left(\alpha_{i}\right)$ is close to $\partial_{i}$ for $i=1,2$ and $o(\beta) \subseteq A\left(\operatorname{graph}\left(\alpha_{1}\right)\right.$, graph $\left.\left(\alpha_{2}\right)\right)$. Let $\phi_{1}$ and $\phi_{2}$ be vertical pushes downwards from graph $\left(\alpha_{1}\right)$ to $\partial_{1}$ and from $\partial_{2}$ to graph $\left(\alpha_{2}\right)$ respectively. If we let $f=\phi_{1} \circ g^{\circ} \phi_{2}$, then $f$ will be monotone twist, and if the pushes $\phi_{1}$ and $\phi_{2}$ are strong enough, using the transit orbit, $f$ will have a non-separating $\Lambda(c)$ for some component $c$. Using the results of $\S 4$ below and the fact that $o(\beta)$ is also an orbit of $f$, we may find a component $c$ that is a non-trivial interval. An interesting question is whether a $\Lambda(\omega)$ for an irrational $\omega$ can be non-separating (cf. [23]).

There are certain cases when $\Lambda(c)$ must separate. For example, if $\Lambda(c)$ is an attractor or repeller or the intersection of attractors or the intersection of repellers (i.e. a quasi-attractor or quasi-repeller), then it must separate. Note that one can determine whether this is the case using the gap order. As another example, if $c$ is a component of $\rho(f)$ with the property that every neighbourhood of the form [ $\sup c, \varepsilon$ ) for $\varepsilon>0$ contains both up and down gaps, then the Aubry-Mather set $\Sigma_{+} \subseteq \Lambda_{+}(c)$ will be an invariant circle so $\Lambda(c)$ will separate. This follows from [8] and [14] since in this case each point in $(\Lambda(c))^{c}$ will be both right and left accessible.

The example in figure 6 shows that a $\Lambda(c)$ may contain gradient-like points. One may also construct such examples for separating $\Lambda(c)$ as follows. We use example 2.1 and let $[a, b]=[-1,1]$ and $T_{1}$ be as in that example. Fix a $p / q$ and for each fixed $y \in\{-1,1\}$ let $\alpha(\cdot, y)$ be a homeomorphism of $\mathbf{R}$ such that $\alpha(x+1, y)=$ $\alpha(x, y)+1, \rho(\alpha(\cdot, y))=p / q$, and if $y_{1}<y_{2}, \alpha\left(x, y_{1}\right)<\alpha\left(x, y_{2}\right)$ for all $x \in \mathbf{R}$. Now define $S^{\prime}:[-1,1] \times \mathbf{R} \rightarrow[-1,1] \times \mathbf{R}$ via $S^{\prime}(x, y)=(\alpha(x, y), y)$ and let $F=S^{\prime} \circ T_{1}$. This $F$ will be monotone twist, $\rho(F)=p / q$ and each point in the interior of the annulus will be gradient-like for $f$. One can clearly embed this construction in a larger annulus to get the desired example.

Although each point in $\Lambda(c)$ need not be chain recurrent, each $\Lambda(c)$ does contain chain transitive sets because, using theorem 2.2, for each $r \in c$ one has an AubryMather minimal set with that rotation number, which of necessity must be contained in $\Lambda(c)$. We also note that although $\Lambda(c)$ is defined using the asymptotic behaviour of orbits, theorem $3.3(\mathrm{~b})$ gives conditions that must be satified by points in $\Lambda(\mathrm{c})$ under a finite number of iterations. This is typical of conclusions resulting from the positive tilt hypothesis (see the remarks after theorem 2.3).

Finally, we give a modification of example 2.1 that shows that any compact set with any gap order can occur as a rotation set for a monotone twist $f$. Given a compact set $K$ and an orientation on its complementary gaps, referring to example 2.1 , let $[a, b]=[\inf K$, sup $K]$ and define a smooth $\phi:[a, b] \rightarrow[a, b]$ so that $\phi(x)>x$ if $x$ is in an up gap, $\phi(x)<x$ if $x$ is in a down gap and $\phi(x)=x$ if $x \in K$. Then $G=S \circ T$ will be the desired example. 


\section{Applications}

In this section we give some applications of our previous results. The Aubry-Mather theorem states that for area-preserving monotone twist $f$, for each $r \in \rho(f)$ one has an Aubry-Mather set with rotation number $r$, and $\rho(f)=$ twist $(f)$. Our first application gives some alternative hypotheses under which this is true. The basic idea is that if $f \in P T$ and $\rho(f) \neq$ twist $(f)$, then $\rho(f)$ has a complementary gap $J$ and so, by theorem 3.3, $f$ has some gradient-like behaviour. Thus if $f$ is in any sense transitive, one must have $\rho(f)=$ twist $(f)$.

Our second application involves a kind of local twist interval associated with an invariant set called its rotation band. Given an $f$-invariant set $Z$, define $H_{-}, H_{+}: \mathbf{R} \rightarrow \mathbf{R}$ via

$$
H_{+}(x)=\sup \left\{\pi_{1}(F(z)): z \in \tilde{Z} \text { and } \pi_{1}(z) \leq x\right\}
$$

and

$$
H_{-}(x)=\inf \left\{\pi_{1}(F(z)): z \in \tilde{Z} \text { and } \pi_{1}(z) \geq x\right\} .
$$

It is clear that $H_{+}$and $H_{-}$are non-decreasing and $H_{+}(x+1)=H_{+}(x)+1$ and so $\rho\left(H_{+}\right)$and $\rho\left(H_{-}\right)$(defined in the usual way) are each a point. We may therefore define the rotation band of $Z$ as $R B(Z)=\left[\rho\left(H_{-}\right), \rho\left(H_{+}\right)\right]$. It is clear that in some sense the rotation band measures the fastest and slowest rates of rotation associated with $Z$ even though there may not exist any orbit in $Z$ with rotation number $\rho\left(H_{-}\right)$ or $\rho\left(H_{+}\right)$. In particular, $\rho(Z)$ may be properly contained in $R B(Z)$. Note that if $Z$ is link-monotone, $R B(Z)=\rho(Z)$. The rotation band is related to the speed interval recently considered in [28] and [4]. It may also be viewed as a generalization of Birkhoff's definition of an inner and outer rotation number for the Birkhoff attractor [8]. As an example, in the twist map induced by the billiard table shown in figure 7 we show a periodic orbit $\beta$. It was shown in [12] that $R B(\beta)=\left[\frac{1}{3}, \frac{1}{2}\right]$ and clearly $\rho(\beta)=\frac{2}{5}$. This is a general phenomenon. If $\beta$ is a $p / q$-periodic orbit that is not monotone and $(p, q)=1$, then $\left[p_{n-1} / q_{n-1}, p_{n} / q_{n}\right] \subseteq R B(\beta)$, where $p_{n-1} / q_{n-1}$ and $p_{n} / q_{n}$ are the last two convergents in the continued fraction. This is true primarily because the rotation band of a periodic orbit depends only on the order structure of the orbit around the annulus.

In corollary 4.1 we show that whenever $Z$ is chain transitive, $R B(Z) \subseteq \rho(f)$. Thus, for example, any positive tilt map which has a periodic orbit with the same order structure as $\beta$ in figure 7 also must have Aubry-Mather sets with rotation number $r$ for each $r$ contained in $\left[\frac{1}{3}, \frac{1}{2}\right]$. In [11] we give a technique for estimating the rotation band of ' $n$-fold' Denjoy minimal sets of the type constructed in [30].

Our third application is closely related to the second and concerns the notion of linked invariant sets. If $f: A \rightarrow A$ is a homeomorphism and $Z_{1}$ and $Z_{2}$ are disjoint invariant sets, we say that $Z_{1}$ and $Z_{2}$ are unlinked if there is a homotopically non-trivial circle $\Gamma$ with $Z_{1} \subseteq A\left(\Gamma, \partial_{1}\right)$ and $Z_{2} \subseteq A\left(\Gamma, \partial_{2}\right)$ and $f(\Gamma) \simeq \Gamma \operatorname{rel} Z_{1}, Z_{2}$. Corollary 4.1(c) states that if $Z_{1}$ and $Z_{2}$ are chain transitive and are linked, then $\left\langle\boldsymbol{R B}\left(Z_{1}\right) \cup \boldsymbol{R B}\left(Z_{2}\right)\right\rangle \subseteq \rho(f)$. Thus if $f$ has a $\frac{1}{5}$ - and a $\frac{1}{7}$-periodic orbit, and each is monotone but the two are linked, then $\left\langle\frac{1}{7}, \frac{1}{5}\right\rangle \subseteq \rho(f)$. 


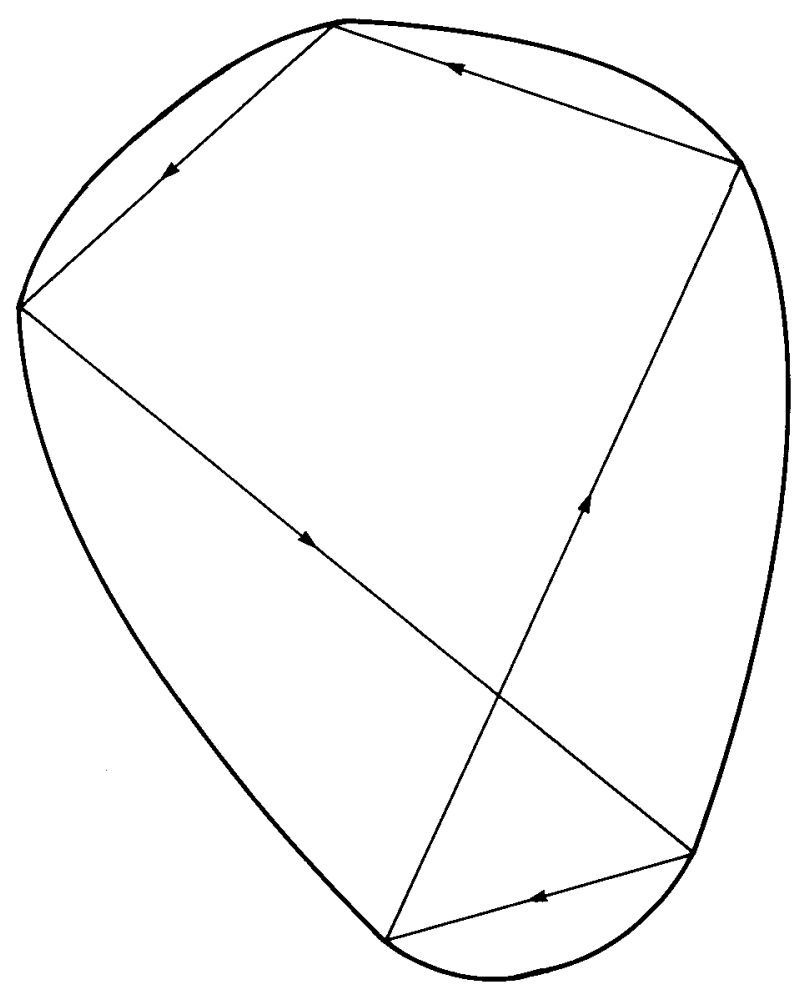

Figure 7

Before stating our corollary we note that most of the results in (a) are essentially known. The case when $f$ is chain transitive follows easily by combining Franks [19], Katok [26] and Hall [20]. Bernstein [5] shows this result for monotone twist $f$ with the graph intersection property (a weaker condition than the circle intersection property). The case when $f$ is area-preserving follows from Hall [21]. Recall that the circle intersection property and the boundary transit property are defined after lemma 3.2 and theorem 3.3 respectively. We include these results to illustrate our techniques.

Corollary 4.1. Let $f \in P T$.

(a) If $f$ is area-preserving, chain transitive, has the circle intersection property or the boundary transit property, then $\rho(f)=$ twist $(f)$.

(b) If $Z$ is a chain transitive set, then $\operatorname{RB}(Z) \subseteq \rho(f)$.

(c) If $Z_{1}$ and $Z_{2}$ are linked chain transitive sets, then

$$
\left\langle R B\left(Z_{1}\right) \cup R B\left(Z_{2}\right)\right\rangle \subseteq \rho(f) .
$$

Proof. If $\rho(f) \neq$ twist $f$, then since $\rho(f) \subseteq$ twist $(f)$, there exists a complementary gap $J$ for $\rho(f)$. Thus using lemma 3.2, there exists a homotopically non-trivial circle $\Gamma$ with $f(\Gamma) \subseteq$ Int $\left(\Gamma, \partial_{i}\right)$, where $i=1$ or 2 depending on whether $J$ is a down gap or up gap respectively. From this it is clear that $f$ cannot have any of the properties given in (a). 
To prove (b), since $Z$ is chain transitive, then by theorem $3.3, Z \subseteq \Lambda(c)$ for some component $c$ of $\rho(f)$. But then $R B(Z) \subseteq R B(\Lambda(c))$ and theorem 3.3(c) makes it clear that $R B(\Lambda(c))=c$.

For (c), note that if $Z_{1} \subseteq \Lambda\left(c_{1}\right)$ and $Z_{2} \subseteq \Lambda\left(c_{2}\right)$ and $c_{1} \neq c_{2}$, then there would be a gap $J$ between $c_{1}$ and $c_{2}$, and thus using theorem 3.3, there is a circle $\Gamma(J)$ with $f(\Gamma(J)) \simeq \Gamma(J)$ is the complement of $\Lambda\left(c_{1}\right)$ and $\Lambda\left(c_{2}\right)$, which is impossible since $Z_{1}$ and $Z_{2}$ are linked. Thus for some $c, Z_{1} \cup Z_{2} \subseteq \Lambda(c)$ and so $\left\langle R B\left(Z_{1}\right) \cup R B\left(Z_{2}\right)\right\rangle \subseteq$ $R B(\Lambda(c))=c$.

We are now in a position to complete the proof of theorem 2.2. For this we need to show that corollary 4.1(b) can be derived independently of what we need to still show, i.e. that if $\alpha \in \rho(f)$ and $\alpha \notin \mathrm{Cl}(\rho(f) \cap \mathbf{Q})$, then $\alpha \in \rho_{\text {mon }}(f)$. Up to this point in the proof of theorem 2.2 we have that $\mathrm{Cl}(\rho(f) \cap Q) \subseteq \rho_{\text {mon }}(f) \subseteq \rho(f)$. Thus if there is a chain transitive $Z$ with $R B(Z)$ not in $\rho(f)$, then there is a rational $p / q \in R B(Z)-\rho(f)$. Now let $G=T^{-p} F^{q}$ and apply theorem 2.3 to $G$ and as in lemma 3.3, $L^{\infty}(G)$ and $R^{\infty}(G)$ are therefore an attractor-repeller pair for $f$ and so $Z \subseteq L^{\infty}(G)$ or $Z \subseteq R^{\infty}(G)$. Applying lemma 3.1 to these two sets shows that $R B\left(R^{\infty}(G)\right) \subseteq\left(p / q, \rho\left(\partial_{2}\right)\right]$ and $R B\left(L^{\infty}(G)\right) \subseteq\left[\rho\left(\partial_{1}\right), p / q\right)$, a contradiction. We now proceed with the proof of theorem 2.2.

Completion of the proof of theorem 2.2. We recall the situation. Since $\rho(\omega(x))=\alpha$, we may choose $z_{0} \in \omega(x)$ with $\rho\left(z_{0}\right)=\alpha$ and $z_{0}$ is non-wandering. Given this, we wish to show that $\alpha \in \rho_{\text {mon }}(f)$. Using the $C^{1}$-closing lemma [32], find diffeomorphisms $f_{k}$ with periodic orbits $z_{k}$ with $z_{k} \rightarrow z_{0}$ and $f_{k} \rightarrow f$ in the $C^{1}$-topology, such that

$$
\left|\pi_{1}\left(F^{k}\left(z_{0}\right)\right)-\pi_{1}\left(z_{0}\right)-\left(\pi_{1}\left(F_{k}^{k}\left(z_{k}\right)\right)-\pi_{1}\left(z_{k}\right)\right)\right|<1
$$

Assume $z_{k}$ is $p_{k} / q_{k}$-periodic. If $\alpha \in \mathrm{Cl}\left(\left\{p_{k} / q_{k}\right\}\right)$, we are done by our two observations at the beginning of the proof so assume there exists a subsequence $p_{k_{i}} / q_{k_{i}} \rightarrow \beta$ with $\beta<\alpha$ (the case $\beta>\alpha$ is similar). For simplicity of exposition, we assume that $p_{k} / q_{k} \rightarrow \beta$ as passing to a subsequence does not alter the proof. By $(*)$, since $\rho\left(z_{0}\right)=\omega$, we have

$$
\pi_{1}\left(F_{k}^{k}\left(z_{k}\right)\right)-\pi_{1}\left(z_{k}\right)=\omega k+r(k)
$$

with $r(k) / k \rightarrow 0$ as $k \rightarrow \infty$. Now for each $k$ find an integer $n(k)$ so that $n(k) q_{k}>k$ and let $p_{k}^{\prime}=n(k) p_{k}$ and $q_{k}^{\prime}=n(k) q_{k}$. Since $z_{k}$ is $p_{k} / q_{k}$-periodic,

$$
p_{k}^{\prime}=\pi_{1}\left(F_{k}^{q_{k}^{\prime}-k}\left(F_{k}^{k}\left(z_{k}\right)\right)\right)-\pi_{1}\left(F_{k}^{k}\left(z_{k}\right)\right)+\pi_{1}\left(F_{k}^{k}\left(x_{k}\right)\right)-\pi_{1}\left(z_{k}\right),
$$

and so if we let $z_{k}^{\prime}=F_{k}^{k}\left(z_{k}\right)$, then

$$
\pi_{1}\left(F_{k}^{q_{k}^{\prime}-k}\left(z_{k}^{\prime}\right)\right)-\pi_{1}\left(z_{k}\right)=p_{k}^{\prime}-\omega k-r(k) .
$$

We therefore have for large enough $k$,

$$
\pi_{1}\left(F_{k}^{k}\left(z_{k}\right)\right)-\pi_{1}\left(z_{k}\right)>[\omega k]-1
$$

and

$$
\pi_{1}\left(F_{k}^{q_{i}^{-k}}\left(z_{k}^{\prime}\right)\right)-\pi_{1}\left(z_{k}^{\prime}\right)<p_{k}^{\prime}-[\omega k]+1,
$$

where for $r \in \mathbf{R},[r]$ denotes the integer part of $r$. Since $z_{k}$ and $z_{k}^{\prime}$ are elements of the same periodic orbit, using what we showed above independently of the result 
we are proving here, we get

where

$$
\left[a_{k}, b_{k}\right] \subseteq \rho\left(f_{k}\right)
$$

$$
a_{k}=\frac{p_{k}^{\prime}-[\omega k]+1}{q_{k}^{\prime}-k}
$$

and

$$
b_{k}=\frac{[\omega k]-1}{k}
$$

Now for large $k, a(k)<b(k)$ and $b(k) \rightarrow \alpha$ and so we may choose rationals $r_{k} \in\left[a_{k}, b_{k}\right]$ with $r_{k} \in \rho\left(f_{k}\right)$ and $r_{k} \rightarrow \alpha$, and since $f_{k} \rightarrow f$ using our two observations, $\alpha \in \rho_{\text {mon }}(f)$.

Our last result gives some simple continuity properties of the rotation set as a function from $P T$ to the closed subsets of $\mathbf{R}$ with the Hausdorff topology, denoted $H(\mathbf{R})$. In [15] Choquet defines upper semicontinuity for these types of maps and shows they are continuous at generic points in the domain (under suitable hypotheses which are satisfied here). We prove upper semicontinuity here, and rather than appeal to this general result we specify (in the proof) a $C^{1}$-generic subset where $\rho$ is continuous.

Note that this upper semicontinuity result is what one expects as a consequence of the stability of the Morse decompositions given in theorem 3.3(a), although we do not use this in our proof.

THEOREM 4.2. If $f \in P T$ and $U \subseteq R$ is an open set with $\rho(f) \subseteq U$, then there exists a $\delta>0$ so that $d(f, g)<\delta$ in the $C^{1}$-topology implies that $\rho(g) \subseteq U$. In addition, there is a generic subset of $P T$ where $\rho: P T \rightarrow H(R)$ is continuous.

Proof. Given $f$, pick an interval $[a, b]$ so that $d(f, g)<1$ in $P T$ implies that $\rho(g) \subseteq$ $[a, b]$. This is possible because $f$ restricted to the boundary circles is a circle homeomorphism which varies continuously with $f[1]$ and so twist $(f)$ varies continuously. If the first statement of the theorem is false, we may find an $r_{k}$ in the compact set $[a, b]-U$ and $f_{k}$ with $d\left(f_{k}, f\right)<1 / k$ with $r_{k} \in \rho\left(f_{k}\right)$. Thus there is a subsequence $r_{k_{i}} \rightarrow r_{0} \in[a, b]-U$ and $f_{k_{i}} \rightarrow f$, and using theorem 2.2 and the first observation of its proof, one has $r_{0} \in \rho(f)$, a contradiction.

Now let $f$ be such that its periodic orbits are dense in the non-wandering set and all periodic orbits of $f$ are hyperbolic. These two properties together are generic for diffeomorphisms by Pugh's density theorem [32] and the Kupka-Smale theorem. Since $P T$ is open in $\operatorname{Diff}_{1}(A)$, they are generic in $P T$ as well.

To show that $\rho$ is continuous at $f$, given $\varepsilon>0$, we must find a $\delta>0$ so that $d(f, g)<\delta$ in the $C^{1}$-topology implies that $\rho(f) \subseteq N_{\varepsilon}(\rho(g))$ and $\rho(g) \subseteq N_{F}(\rho(f))$, where for a set $X \subseteq \mathbf{R}, N_{\varepsilon}(X)=\{y: d(y, x)<\varepsilon$ for some $x \in X\}$. The second inclusion follows from what we have just proved. For the first inclusion we shall need the fact that for generic $f$ as above, $\rho \overline{(f) \cap \mathbf{Q}}=\rho(f)$. Assume this is false, then

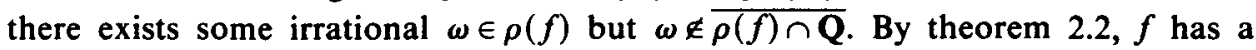
Denjoy minimal set or an invariant circle $\Sigma$ with $\rho(\Sigma)=\omega$. Now we are assuming 
that periodic orbits are dense in the non-wandering set and certainly $\Sigma$ must contain a non-wandering point $x$ and so we may find periodic orbits $z_{i} \rightarrow z$. An argument similar to that given in the continuation of the proof of theorem 2.2 given above then implies that $\omega \in \overline{\rho(f) \cap \mathbf{Q} \text {. }}$

We therefore have that for all $\varepsilon>0, \cup\left\{N_{\varepsilon}(p / q): p / q \in \mathbf{Q} \cap \rho(f)\right\}$ covers $\rho(f)$. Using the compactness of $\rho(f)$, find a finite set with $\rho(f) \subseteq \bigcup_{i=1}^{k} N_{\varepsilon}\left(p_{i} / q_{i}\right)$. Now by theorem 2.2 we may find a $p_{i} / q_{i}$-periodic orbit $z_{i}$ for $i=1, \ldots, k$. In addition, these are hyperbolic by our assumptions on $f$ and so we may find a $\delta$ so that $d(f, g)<\delta$ in the $C^{1}$-topology implies that $z_{i}$ is a $p_{i} / q_{i}$-periodic orbit for $g$. Thus $\left\{p_{i} / q_{i}\right\} \subseteq \rho(g)$ and so $\rho(f) \subseteq \bigcup_{i=0}^{k} N_{\varepsilon}\left(p_{i} / q_{i}\right) \subseteq N_{\varepsilon}(\rho(g))$.

In this proof we have shown that for generic $f, \rho(f)=\overline{\rho(f) \cap \mathbf{Q}}$, which implies that generically $\rho(f)$ has a countable number of components. Question: does $\rho(f)$ generically have a finite number of components?

We also note that $\rho(f)$ will not be continuous in generic one-parameter families. For example, let $F_{\mu}$ be fixed point free for $\mu<0$ with $\rho\left(F_{\mu}\right)=\{-1,1\}$ say. At $\mu=0$ let there be a saddle node bifurcation to create a fixed sink-saddle pair for $\boldsymbol{\mu}>0$. Then any family close to $F_{\mu}$ will also have the discontinuous transition of rotation sets from $\{-1,1\}$ to $\{-1,0,1\}$ (or something larger).

\section{REFERENCES}

[1] V. I. Arnol'd. Small denominators I; On the mappings of the circumference onto itself. Transl. AMS 46, Series 2 (1965), 213-284.

[2] D. Aronson, M. Chory, G. R. Hall \& R. McGehee. Bifurcation from an invariant circle for two parameter families of maps of the plane: a computer assisted study. Comm. Math. Phys. 83 (1982), 303-354.

[3] S. Aubry \& P. Y. Le Daeron. The discrete Frenkel-Kontorova model and its extensions I. Physica $D 8$ (1983), 381-422.

[4] J. Barkmeijer. The speed interval of maps of the circle. Preprint.

[5] D. Bernstein. Birkhoff periodic orbits for twist maps with the circle intersection property. Ergod. Th. \& Dynam. Sys. 5 (1985), 531-537.

[6] G. D. Birkhoff. Surface transformations and their dynamical applications. Acta Math. 43 (1920), 44-74. Reprinted in Collected Mathematical Papers of G. D. Birkhoff, Vol. II. Dover, New York (1968), 195-202.

[7] G. D. Birkhoff. Dynamical Systems. AMS Colloquium Publications IX. AMS, Providence, RI (1927).

[8] G. D. Birkhoff. Sur quelques courbes fermées remarquables. Bull. SMF 60 (1932). Reprinted in Collected Mathematical Papers of G. D. Birkhoff, Vol. II. Dover, New York (1968), 418-443.

[9] P. Boyland. Bifurcations of circle maps: Arnol'd tongues, bistability and rotation intervals. Comm. Math. Phys. 106 (1986), 353-381.

[10] P. Boyland. Braid types and a topological method of proving positive entropy. Preprint.

[11] P. Boyland. Invariant circles and rotation bands in twist maps. Comm. Math. Phys. 113 (1987), 67-77.

[12] P. Boyland \& G. R. Hall. Invariant circles and the order structure of periodic orbits in monotone twist maps. Topology 26 (1987), 21-35.

[13] M. Casdagli. Periodic orbits for dissipative twist maps. Ergod. Th. \& Dynam. Sys. 7 (1987), $165-173$.

[14] M. Charpentier. Sur quelques propriétés des courbes de M. Birkhoff. Bull. SMF 62 (1934), 193-224.

[15] G. Choquet. Lectures on Analysis, Vol. 1. Benjamin Inc., New York (1969).

[16] C. Conley. Isolated Invariant Sets and the Morse Index. CBMS Regional Conference Series in Mathematics 38. AMS, Providence, RI (1978).

[17] C. Conley. The gradient structure of a flow: I. Ergod. Th. \& Dynam. Sys. 8* (1988), 11-26.

[18] R. Douady. Application du théorème des tores invariants. Thèse de 3 e cycle. Université Paris VII (1982). 
[19] J. Franks. Recurrence and fixed points of surface homeomorphisms. Ergod. Th. \& Dynam. Sys. 8* (1988), 99-107.

[20] G. R. Hall. A topological version of a theorem of Mather on twist maps. Ergod. Th. \& Dynam. Sys. 4 (1984), 585-603.

[21] G. R. Hall. Birkhoff orbits for non-monotone twist maps. Preprint.

[22] M. Handel. Zero entropy surface diffeomorphisms. Preprint.

[23] J. Harrison. $C^{2}$-counterexample to the Seifert conjecture. Preprint.

[24] M. Herman. Sur les courbes invariantes par les difféomorphismes de l'anneau. Astérisque 103-104 (1983).

[25] K. Hockett \& P. Holmes. Josephson's junction, annulus maps, Birkhoff attractors, horseshoes and rotation sets. Ergod. Th. \& Dynam. Sys. 6 (1986), 205-211.

[26] A. Katok. Some remarks on Birkhoff and Mather twist map theorems. Ergod. Th. \& Dynam. Sys. 2 (1982), 185-192.

[27] P. Le Calvez. Existence d'orbites quasi-periodiques dans les attracteurs de Birkhoff. Comm. Math. Phys. 106 (1986), 383-394.

[28] R. MacKay. Rotation interval from a time series. J. Phys. A: Math. Gen. 20 (1987), 587-592.

[29] J. Mather. Non-existence of invariant circles. Ergod. Th. \& Dynam. Sys. 4 (1984), 301-309.

[30] J. Mather. More Denjoy minimal sets for area preserving diffeomorphisms. Comm. Math. Helv. 60 (1985), 508-557.

[31] R. Moeckel. Morse decompositions and connection matrices. Ergod. Th. \& Dynam. Sys. 8* (1988), 227-249.

[32] C. Pugh. An improved closing lemma and a general density theorem. Am. J. Math. 89 (1967), 1010-1021.

[33] J. C. Yoccoz. Bifurcations de points fixes elliptiques (d'après A. Chenciner). Astérisque 145-146 (1987), 313-334. 